\title{
SPECTRAL BOUNDARY-VALUE PROBLEMS FOR THE DIRAC SYSTEM WITH A SINGULAR POTENTIAL
}

\author{
M. S. AGRANOVICH AND G. ROZENBLUM
}

Dedicated to Mikhail Shlemovich Birman on the occasion of his 75th birthday

\section{Introduction. SETting OF THE PROBLEMS}

We write the free Dirac operator in $\mathbb{R}^{3}$ as

$$
\mathcal{D}=\sum_{1}^{3} \alpha_{j} D_{j}+\beta
$$

Here $D_{j}=-i \partial_{j}=-i \partial / \partial x_{j}, \alpha_{j}$ are Hermitian $(4 \times 4)$-matrices

$$
\alpha_{j}=\left(\begin{array}{cc}
0 & \sigma_{j} \\
\sigma_{j} & 0
\end{array}\right),
$$

where $\sigma_{j}$ are the Pauli matrices

$$
\sigma_{1}=\left(\begin{array}{ll}
0 & 1 \\
1 & 0
\end{array}\right), \quad \sigma_{2}=\left(\begin{array}{cc}
0 & -i \\
i & 0
\end{array}\right), \quad \sigma_{3}=\left(\begin{array}{cc}
1 & 0 \\
0 & -1
\end{array}\right),
$$

and $\beta=\alpha_{4}$ is the diagonal $(4 \times 4)$-matrix with the main diagonal $(1,1,-1,-1)$. These matrices have eigenvalues \pm 1 and satisfy the relations

$$
\begin{array}{cll}
\sigma_{l} \sigma_{k}=-\sigma_{k} \sigma_{l} & (l \neq k), \quad \sigma_{l}^{2}=I_{2} ; \\
\alpha_{l} \alpha_{k}=-\alpha_{k} \alpha_{l} \quad(l \neq k), & \alpha_{l}^{2}=I_{4} .
\end{array}
$$

Here and in what follows $I_{m}$ is the identity $(m \times m)$-matrix. Vector-valued functions $w(x)$, on which the operator $\mathcal{D}$ acts, will be written in the form

where

$$
w=\left(\begin{array}{l}
u \\
v
\end{array}\right)
$$

We also set

$$
u=\left(\begin{array}{l}
u_{1} \\
u_{2}
\end{array}\right), \quad v=\left(\begin{array}{c}
v_{1} \\
v_{2}
\end{array}\right) .
$$

The square of this matrix is $|\xi|^{2} I_{2}$. The form (1) of the Dirac system was used in [1] and many other papers included in our references.

2000 Mathematics Subject Classification. Primary 35Q40.

Key words and phrases. Dirac operator, Coulomb singularity, boundary-value problems, selfadjoint extensions, behavior of eigenvalues.

The first author was supported by RFBR (grant no. 01-01-00284) and by a grant of the Swedish Research Council. 
Let $\Omega$ be a bounded domain in $\mathbb{R}^{3}$. For simplicity we assume that its boundary $\Gamma$ is infinitely smooth. By $\nu=\nu(x)$ we denote the outward unit normal at the point $x \in \Gamma$. Consider the following boundary-value problem with two real parameters $\lambda$ and $\mu$ :

$$
\begin{aligned}
& (\mathcal{D}+V) w(x)-\lambda w(x)=f(x) \quad(x \in \Omega), \\
& B u:=i \sigma(\nu(x)) v^{+}(x)-\mu u^{+}(x)=g(x) \quad(x \in \Gamma) ;
\end{aligned}
$$

here and below the superscript ${ }^{+}$means the boundary values of functions. By $V(x)$ we denote a Hermitian potential that may have a Coulomb-like singularity at the origin (we assume that it lies inside $\Omega$ ). In the simplest case this singularity has the form $\theta /|x|$, where $\theta$ is a real number. The potential $V(x)$ is the sum of this fraction and a function smooth in $\bar{\Omega}$. It is the presence of the Coulomb singularity that leads to many analytical difficulties we overcome in the paper. In the general case, the potential can be a matrix-valued function subject to some inequalities and additional restrictions.

System (6) corresponds, for example, to an electron in the field of a charged point nucleus. The problem (6), (7) was posed by physicists (see [2] and references therein). However, the boundary condition has no physical meaning and is imposed for calculation purposes. Namely, the domain $\Omega$ is regarded as a "black box", and one intends to find what happens on its boundary. More precisely, let the values of the parameters $\lambda$ and $\mu$ be such that the problem has a unique solution, and let $f=0$. The problem consists in finding $u^{+}$in terms of $g$. The operator mapping $g$ to $u^{+}$is called the $R$-matrix. If $u^{+}$ is found and $\mu$ is different from 0 (this is assumed as well), then $v^{+}$also can be found from the boundary condition (7), so the Cauchy data of the solution are determined completely. For the homogeneous Dirac system these Cauchy data from the inside are the same as those from the outside, and the latter replace the system in $\Omega$ when the problem is considered in the exterior domain $\mathbb{R}^{3} \backslash \bar{\Omega}$.

Two approaches to constructing the $R$-matrix are suggested by physicists. Namely, setting $f=0, g=0$, we get two spectral problems.

I. The problem with $\lambda$ as a spectral parameter and fixed $\mu$.

II. The problem with $\mu$ as a spectral parameter and fixed $\lambda$.

The approaches consist in using the spectral expansions in eigenfunctions of problems I and II, respectively.

Our aim in the present paper is to investigate spectral properties of both problems and to clarify how to use them rigorously in the mathematical context for constructing the $R$-matrix. Problems I and II are considered in Chapters I and II, respectively.

We were informed about these problems, the notion of the $R$-matrix, and two approaches to its construction by R. Szmytkowski, the author of the book [2]. We owe him our sincere gratitude for this as well as for consultations and a discussion of our results.

Before passing to their brief description, we make two remarks.

Remark 1. At first sight, $u^{+}$and $v^{+}$have different status in the boundary condition. However, in fact, their roles can be interchanged by multiplying the boundary condition by $-i \sigma(\nu) / \mu$ and replacing $\mu$ with $\widetilde{\mu}=-1 / \mu$; cf. [2].

Remark 2. By the well-known Hardy inequality

$$
\int \frac{|h(x)|^{2}}{|x|^{2}} d x \leq 4 \int|\nabla h|^{2} d x \quad\left(h \in C_{0}^{\infty}\left(\mathbb{R}^{3}\right)\right),
$$

where $h$ is a scalar function (see, e.g., 3, Chapter VI, §5]), multiplication by a potential with a Coulomb singularity is a bounded operator from the Sobolev space $H^{1}(\Omega)=$ $W_{2}^{1}(\Omega)$ to $H^{0}(\Omega)=L_{2}(\Omega)$. Indeed, it is well known that the functions in $H^{1}(\Omega)$ can be extended to finitely supported functions in $H^{1}\left(\mathbb{R}^{3}\right)$ by using a bounded extension 
operator. Therefore, the operator $\mathcal{D}+V$ with a Coulomb potential is a bounded operator from $H^{1}(\Omega)^{4}$ to $L_{2}(\Omega)^{4}$.

To a great extent, our investigation is based on fine results that are known for the Dirac operator in $\mathbb{R}^{3}$ with a Coulomb-type potential. However, taken alone, these results are insufficient, and we need to consider some specific questions, starting with the definition and description of the selfadjoint operators that correspond to problems I and II and concluding with the investigation of the behavior of their eigenvalues.

We begin with the explanation of the contents of Chapter I.

When the potential $V$ does not involve a singularity, the problem (6), (7) with a nonzero real $\mu$ is elliptic, and integration by parts readily shows that it is formally selfadjoint (see [4] 1 ). This leads immediately to the following description of the properties of the first spectral problem. The differential operation $\mathcal{D}+V$ and the boundary condition determine a selfadjoint operator $(\mathcal{D}+V)_{\Omega}$ in $L_{2}(\Omega)^{4}$. Its domain is a subspace of the Sobolev $L_{2}$-space $H^{1}(\Omega)^{4}$; this subspace consists of all functions satisfying the homogeneous boundary condition (7) with $g=0$ and is denoted by $H^{1}(\Omega, B)^{4}$. The operator $(\mathcal{D}+V)_{\Omega}$ acts boundedly from this subspace to $L_{2}(\Omega)^{4}$. The spectrum of this operator is discrete, there exists an orthonormal basis of eigenfunctions in $L_{2}(\Omega)^{4}$, and the eigenvalues have the usual asymptotic behavior

$$
\lambda_{n}=b n^{1 / 3}+O(1) \quad(n \rightarrow \pm \infty), \quad b=\left(\frac{3 \pi^{2}}{\operatorname{meas} \Omega}\right)^{1 / 3} .
$$

Here the positive and negative eigenvalues are numbered in a nondecreasing way, counting their multiplicities. The remainder estimate is the best possible. The operator is not semibounded, and thus the eigenvalues have two limit points $\pm \infty$. The orthonormal basis of eigenfunctions in $L_{2}(\Omega)^{4}$ remains to be an unconditional basis in $H^{1}(\Omega, B)^{4}$. (A basis is called unconditional if it remains to be a basis after any permutation of its terms.)

As to formula (9), we refer the reader to the general results of [5 $\S 6]$. There is another way to deduce this asymptotics, however without an efficient estimate of the remainder. For $V=0$ it consists in passing to the variational problem for the second order system $\mathcal{D}^{2} w=\lambda \mathcal{D} w$ and then using the results of [6] for the corresponding ratio of the quadratic forms first on $H^{1}(\Omega)^{4}$ and on $H_{0}^{1}(\Omega)^{4}$, which corresponds to the Neumann and Dirichlet variational problems with homogeneous boundary conditions. In these cases we get one and the same asymptotic behavior; therefore it is extended to our problem as well. The lower order terms in the operator that appear due to the potential do not influence the leading term of the asymptotics.

In the case of a potential with singularity, we can no longer use the results of the theory of elliptic boundary-value problems directly.

But in this case, starting from the 1970s, the problem of constructing and describing a selfadjoint operator in $\mathbb{R}^{3}$ that corresponds to the Dirac operator with a potential having a Coulomb singularity has been studied actively. The history of this problem and detailed references can be found in [7, Chapter 4, pp. 305-306]. We also mention the survey [8]. If, for simplicity, the potential is scalar, then three cases are distinguished:

1) $|\theta|<\sqrt{3} / 2$,

2) $\sqrt{3} / 2 \leq|\theta|<1$

3) $|\theta| \geq 1$.

The growth of $|\theta|$ corresponds to that of the "atomic number" of the nucleus. In the first case the atomic numbers are less than 119, in the second they are between 119 and 137. These cases are most interesting for the atomic physics. In the first case it is proved that the Dirac operator in $C_{0}^{\infty}\left(\mathbb{R}^{3} \backslash\{0\}\right)^{4}$ is essentially selfadjoint, i.e., the closure of

\footnotetext{
${ }^{1}$ Our notation is slightly different from that in [4].
} 
this operator in $L^{2}\left(\mathbb{R}^{3}\right)^{4}$ is selfadjoint. For simplicity, let the potential be compactly supported; then the domain of this closure is the space $H^{1}\left(\mathbb{R}^{3}\right)^{4}$. In the second case there are infinitely many selfadjoint extensions; however, a distinguished one can be constructed corresponding to finite kinetic and potential energy states. Now the domain consists of the functions $w \in H^{1 / 2}\left(\mathbb{R}^{3}\right)^{4}$ such that $\varphi w \in H^{1}\left(\mathbb{R}^{3}\right)^{4}$ for smooth functions $\varphi$ vanishing near the origin and bounded together with the first derivatives. The reason of an abrupt smoothness loss near the singularity is clearly seen for the pure Coulomb potential $V(x)=\theta /|x|$ if the required operator is constructed by separation of variables (we briefly dwell on this in $\S 5$, where separation of variables for the boundary-value problem will be performed). In $\S 1$ and $\S 2$ the results pertaining to the construction of this operator are described in more detail. Subsequently, these results will be used in an essential way.

In the third case, for large atomic numbers, presumably there is no physical reason to single out a distinguished selfadjoint extension. Moreover, it is believed that the Dirac-Coulomb system is not quite adequate to the physical situation for heavy nuclei. Nevertheless, there are some publications concerning this case as well.

In $\S \S 1$ and 2, we associate a selfadjoint operator $(\mathcal{D}+V)_{\Omega}$ in $L_{2}(\Omega)^{4}$ with the problem (6), (7) for $g=0$, describe its domain, its action on functions in the domain, and spectral properties of this operator. Cases 1) and 2) will be considered in $\S 1$ and $\S 2$, respectively, and we shall "glue together" the results for the Dirac system with a singular potential in the entire space and the results for the boundary-value problem without singularities.

In the first case the domain of this operator is the space $H^{1}(\Omega, B)^{4}$. The second case is somewhat more complicated and the domain is "worse" in this case: near the singularity the functions belonging to the domain are in $H^{1 / 2}$ only, so that a special definition of the norm in this space is required in order to ensure the boundedness of the direct and inverse operators (outside the spectrum). In fact this is the graph norm for this operator.

In both cases the spectrum of our operator turns out to be discrete. The eigenfunctions still form a basis in the domain of the operator. In $\S 3$ we establish the usual asymptotic behavior for the eigenvalues, as in (9), with the same limit points $\pm \infty$ and the same constant $b$ (but without an efficient remainder estimate).

However, the latter is not quite simple. The point is that the term with singular potential is not relatively compact with respect to the rest of the operator. We overcome this difficulty by considering the difference of resolvents for the perturbed and nonperturbed operators. Here the results by Nenciu [9] are essentially used, namely, the abstract approach for nonsemibounded operators with the study of the resolvent, and realization of this approach in $\mathbb{R}^{3}$. First we show that the operator we define for our problem is also a realization of the abstract Nenciu approach. This fact is interesting in itself. At the concluding step, we use the estimate of the spectrum proved in [6, 10].

The main results are stated in Theorems 1.1, 2.5, and 3.1. They cover some situations with matrix-valued potentials and can be extended to potentials with Coulomb singularities at several points.

In case 3 ) we only note the possibility to describe all the possible selfadjoint operators that correspond to the problem with pure Coulomb potential in a ball. See $\S 5$.

We turn to the contents of Chapter II. For a smooth potential, the second spectral problem was considered in [4]. Even in that case, the problem is unusual. To simplify the notation, we put $\lambda=0$; but we need to assume that the functions $V(x) \pm 1$ do not vanish anywhere (cf. [11]). Then, excluding $v$ or $u$ from (6), we get strongly elliptic second order $(2 \times 2)$-systems in $\Omega$. Suppose that the corresponding Dirichlet problems have unique solutions. Then, by using the corresponding Poisson operators, it is possible to reduce 
the spectral problem II to a spectral equation in $L_{2}(\Gamma)^{2}$ with respect to $u^{+}$(or to $v^{+}$) with a first order pseudodifferential operator. This operator turns out to be nonelliptic but invertible on the set of infinitely smooth functions. Actually, the nonellipticity of this operator is related to the fact that the Dirichlet problem for the Dirac system (where either $u^{+}$or $v^{+}$is given) is nonelliptic; cf. [11]. Furthermore, it turns out that the linear combinations of this operator and its inverse with two nonzero coefficients are elliptic. (In 4 only one linear combination was considered, which is insufficient in the case of variable coefficients.) This leads to the existence of a smooth orthonormal basis in $L_{2}(\Gamma)^{2}$ consisting of the parts $u_{n}^{+}$of eigenfunctions on $\Gamma$ with two limit points for the eigenvalues, namely, 0 and $\infty$ (more precisely, +0 and $-\infty$ ), which is rather unusual. Moreover, the asymptotic behavior of both eigenvalue sequences is given with remainder estimates. It is possible to form a basis consisting of the lower parts $v_{n}^{+}$of the eigenfunctions as well. The property to be a basis persists for all Sobolev spaces on $\Gamma$.

We extend this result to the case of potentials with Coulomb singularity for $0<|\theta|<1$. To do this, we again consider the corresponding second order systems. However, now they are degenerate in its principal term and have a Coulomb singularity in the lower order term. Roughly speaking, they are systems of the following structure:

$$
\nabla|x| \nabla u(x)+\frac{\theta^{2}}{|x|} u(x)=0 .
$$

It is possible to handle the corresponding Dirichlet problem by using some special analogs of the Hardy and Gårding inequalities. For a scalar system like (10), the required analog of the Hardy inequality is the following:

$$
\int \frac{|h(x)|^{2}}{|x|} d x \leq \int|x||\nabla h(x)|^{2} d x, \quad h \in C_{0}^{\infty}\left(\mathbb{R}^{3}\right)
$$

(see [13]). This inequality can be verified in the same way as the standard inequality (8) (see [3]) but with the substitution $w=r h$ instead of $w=r^{1 / 2} h$, where $r=|x|$.

We shall need a similar inequality with $\sigma(D)$ instead of $\nabla$ (for two-dimensional vectorvalued functions) actually contained in [13]; cf. also [12]. Under the condition that the Dirichlet problems mentioned above are solvable, the spectral results remain true without losses. They are stated in Theorem 10.1.

In $\S \S 4$ and 11 we discuss two spectral methods of constructing the $R$-matrix for the Dirac system.

First, we note that for the Schrödinger equation, in the case of unique solvability of the (scalar) problem

$$
\Delta u+V u-\lambda u=0 \text { in } \Omega, \quad \partial_{\nu} u-\mu u=g \text { on } \Gamma
$$

(here $\partial_{\nu}$ is the normal derivative), the operator $g \mapsto u^{+}$is called the $R$-matrix. This operator can be constructed either by using the eigenfunctions of the problem with spectral parameter $\lambda$ in $\Omega$, or by using the eigenfunctions of the problem with spectral parameter $\mu$ on $\Gamma$; see [2] and references therein. In the atomic and molecular physics, this notion of the $R$-matrix for the Schrödinger equation and the spectral approaches to its construction seem to be very popular. They were analyzed by mathematical tools in [14, 15].

For the Dirac system, the notion of the $R$-matrix and attempts to use the spectral approaches for its construction appeared by a natural analogy (again see [2] and the references therein). We have not succeeded in justification of the approach that involves the eigenfunctions of problem I; however, we propose a satisfactory (from our point of 
view) regularization of the corresponding series. As to the approach based on the eigenfunctions of problem II, it admits a complete and convenient justification. Apparently, the first physical paper in this direction was [16] (see also [2] and references therein).

Concluding this Introduction, we would like to mention a continuous and salutary effect of the remarkable investigations, ideas, and results of M. Sh. Birman on our mathematical work. On the occasion of his jubilee birthday, we wish him good health and a successful continuation of his creative activity during many years.

We agree to identify a function defined in $\Omega$ and vanishing near $\Gamma$ with the function in $\mathbb{R}^{3}$ that extends the original one by zero outside $\Omega$.

The symbol $O_{\varepsilon}$ will denote the open ball of radius $\varepsilon$ with center at the origin. For $\varepsilon$ sufficiently small, its closure is contained in $\Omega$, and then we use the notation $\Omega_{\varepsilon}=\Omega \backslash \bar{O}_{\varepsilon}$. We also put $\Omega_{0}=\Omega \backslash\{0\}$ and $\mathbb{R}_{0}^{3}=\mathbb{R}^{3} \backslash\{0\}$.

Positive constants $C_{j}$ do not depend on the functions under consideration, and usually they are numbered anew in each new section.

\section{Chapter I. THE PROBLEM WITH SPECTRAL PARAMETER IN THE SYSTEM}

\section{$\S 1$. The Case of a Coulomb singularity with $|\theta|<\sqrt{3} / 2$}

In this section we assume that

$$
V(x)=V_{0}(x)+V_{1}(x),
$$

where $V_{1}(x)$ is a smooth $\left(C^{1}\right)$ function in $\bar{\Omega}$ and $V_{0}(x)$ is a function smooth everywhere except the origin, vanishing near $\Gamma$, and satisfying the inequality

$$
\left|V_{0}(x)\right| \leq \frac{\theta}{|x|}, \quad 0 \leq \theta<\frac{\sqrt{3}}{2} .
$$

Both functions may be Hermitian matrices, and then inequality (1.2) (as well as similar inequalities below) is related to the operator norm of the matrix, which is equal to the maximum modulus of the eigenvalues. In addition, as in [13, we assume that the matrix $V_{0}(x)$ commutes with the matrix $x \cdot \alpha=\sum_{1}^{3} x_{j} \alpha_{j}$. Let $V(x)$ be of the same form in $\mathbb{R}^{3}$ with compactly supported $V_{j}(x)$.

In this and the next section we denote the Sobolev spaces $H^{s}\left(\mathbb{R}^{3}\right)^{4}$ by $H^{s}$; in particular, $L_{2}\left(\mathbb{R}^{3}\right)^{4}=H^{0}\left(\mathbb{R}^{3}\right)^{4}$ is denoted by $L_{2}$ or $H^{0}$.

The Dirac operator $(\mathcal{D}+V)_{\mathbb{R}^{3}}$ with such a potential was considered by Schminke [17] and later by Vogelsang [13] in the scalar case (under a slightly more general assumptions than ours) and in the matrix case, respectively. Generalizing results of their predecessors, these authors proved that this operator defined on $C_{0}^{\infty}\left(\mathbb{R}_{0}^{3}\right)^{4}$ is essentially selfadjoint in $L_{2}$. As was already mentioned in the Introduction, the domain of the corresponding selfadjoint operator is the space $H^{1}$ if, say, the potential is compactly supported. In fact, its behavior at infinity plays no role for selfadjointness (see [18] and the references therein to the papers by Chernov and other authors).

Recall that in the boundary-value problem (6), (7) the number $\mu$ is assumed to be real and nonzero.

Theorem 1.1. Under the assumptions indicated above, the problem (6), (7) with $\lambda=0$ and $g=0$ determines a selfadjoint operator in $L_{2}(\Omega)^{4}$ with domain $H^{1}(\Omega, B)^{4}$. Its spectrum is discrete. In $L_{2}(\Omega)^{4}$ it has an orthonormal basis of eigenfunctions, which remains to be an unconditional basis in $H^{1}(\Omega, B)^{4}$.

If the function $V(x)$ for $x \neq 0$ and the boundary are infinitely smooth, then the eigenfunctions belong to $C^{\infty}(\bar{\Omega} \backslash\{0\})$. 
Proof. Remark 2 in the Introduction implies that the operator $(\mathcal{D}+V)_{\Omega}$ corresponding to the problem under consideration is always bounded as an operator from $H^{1}(\Omega, B)^{4}$ to $H^{0}(\Omega)^{4}$. First, we check that under our assumptions it is a Fredholm operator. To do this, we establish an a priori estimate and construct a right parametrix (see, e.g., [19]).

Let $\varphi_{1}$ be a (scalar) function in $C_{0}^{\infty}(\Omega)$ that equals 1 in a neighborhood of the origin, and let $\varphi_{2}=1-\varphi_{1}$ in $\bar{\Omega}$. Somewhat later we shall need two more functions: $\psi_{1}$ in $C_{0}^{\infty}(\Omega)$ will be equal to 1 in a neighborhood of $\operatorname{supp} \varphi_{1}$, and $\psi_{2}$ in $C^{\infty}(\bar{\Omega})$ will be equal to 1 in a neighborhood of $\operatorname{supp} \varphi_{2}$ and to 0 in a neighborhood of the origin. All these functions are assumed to be nonnegative.

First, we assume that $V_{1}(x)=0$. Let $w$ be an arbitrary function in $H^{1}(\Omega, B)^{4}$. The following inequality by Vogelsang [13] is true for $\varphi_{1} w$ regarded as a compactly supported function on $\mathbb{R}^{3}$ :

$$
\left\|\varphi_{1} w /|x|\right\|_{0, \mathbb{R}^{3}} \leq C_{1}\left\|(\mathcal{D}+V)\left(\varphi_{1} w\right)\right\|_{0, \mathbb{R}^{3}} .
$$

More precisely, this estimate is proved for $C_{0}^{\infty}\left(\mathbb{R}_{0}^{3}\right)^{4}$-functions, but it is known (see, e.g., [7, p. 12]) that such functions are dense in $H^{1}\left(\mathbb{R}^{3}\right)^{4}$. As a consequence, we obtain the estimate

$$
\left\|\mathcal{D}\left(\varphi_{1} w\right)\right\|_{0, \mathbb{R}^{3}} \leq C_{2}\left\|(\mathcal{D}+V)\left(\varphi_{1} w\right)\right\|_{0, \mathbb{R}^{3}} .
$$

Since the operator $\mathcal{D}$ is elliptic and the function $\mathcal{D} \varphi_{1}$ is bounded, this implies

$$
\left\|\varphi_{1} w\right\|_{1, \Omega} \leq C_{3}\left[\|(\mathcal{D}+V) w\|_{0, \Omega}+\|w\|_{0, \Omega}\right] .
$$

On the other hand, since the problem without singularity is elliptic, we have

$$
\left\|\varphi_{2} w\right\|_{1, \Omega} \leq C_{4}\left[\left\|(\mathcal{D}+V)\left(\varphi_{2} w\right)\right\|_{0, \Omega}+\left\|\varphi_{2} w\right\|_{0, \Omega}\right],
$$

so that

$$
\left\|\varphi_{2} w\right\|_{1, \Omega} \leq C_{5}\left[\|(\mathcal{D}+V) w\|_{0, \Omega}+\|w\|_{0, \Omega}\right] .
$$

From (1.4) and (1.5) we arrive at

$$
\|w\|_{1, \Omega} \leq C_{6}\left[\|(\mathcal{D}+V) w\|_{0, \Omega}+\|w\|_{0, \Omega}\right],
$$

and this is the required estimate. Of course, it can be extended to the case of $V_{1} \neq 0$. Moreover, we can add $i s I_{4}$ to the potential $V$, where we take $s$ real.

Now we construct a right parametrix. Again, let $V_{1}=0$ for the beginning.

Denote by $R_{1}$ the operator inverse to $(\mathcal{D}+V)_{\mathbb{R}^{3}}+i s_{0} I_{4}$ with a nonzero real $s_{0}$. This inverse exists because the operator $(\mathcal{D}+V)_{\mathbb{R}^{3}}$ is selfadjoint and is a bounded operator from $L_{2}\left(\mathbb{R}^{3}\right)^{4}$ to $H^{1}\left(\mathbb{R}^{3}\right)^{4}$. By $R_{2}$ we denote the operator acting from $L_{2}(\Omega)^{4}$ to $H^{1}(\Omega, B)^{4}$ and inverse to the operator corresponding to the boundary problem $(6),(7)$ with $g=0$, the potential $\psi_{2} V$ instead of $V$, and the same additional term $i s_{0} I_{4}$. The operator $R_{2}$ exists because the analogous operator with $s_{0}=0$ is selfadjoint. (In what follows the symbol $I_{4}$ will usually be omitted.) Put

$$
R=\psi_{1} R_{1}\left(\varphi_{1} \cdot\right)+\psi_{2} R_{2}\left(\varphi_{2} \cdot\right)
$$

Let $f$ be a function in $L_{2}(\Omega)^{4}$. The function $u=R f$ satisfies the boundary condition (7) with $g=0$, and

$$
\begin{aligned}
(\mathcal{D}+V)_{\Omega} R f= & \psi_{1}\left(\mathcal{D}+V+i s_{0}-i s_{0}\right) R_{1}\left(\varphi_{1} f\right)+\psi_{2}\left(\mathcal{D}+V+i s_{0}-i s_{0}\right) R_{2}\left(\varphi_{2} f\right) \\
& +\left(\sum_{1}^{3} \alpha_{j} D_{j} \psi_{1}\right) R_{1}\left(\varphi_{1} f\right)+\left(\sum_{1}^{3} \alpha_{j} D_{j} \psi_{2}\right) R_{2}\left(\varphi_{2} f\right) .
\end{aligned}
$$

From this we see that

$$
(\mathcal{D}+V)_{\Omega} R f=f+U f,
$$


where $U$ is a bounded operator from $L_{2}(\Omega)^{4}$ to $H^{1}(\Omega)^{4}$ and hence a compact operator in $L_{2}(\Omega)^{4}$. Consequently, the operator $R$ is a right parametrix we are looking for. It remains to be a right parametrix after adding a smooth function and $i s$ to $V$ (the formula for $R$ does not require any changes).

We have proved that $(\mathcal{D}+V)_{\Omega}$ is a Fredholm operator.

All this remains true if we multiply the potential by $t, 0 \leq t \leq 1$. Since the index does not change under continuous deformations and, obviously, equals zero for $t=0$ (as the index of a formally selfadjoint elliptic problem), it is equal to zero for $t=1$ as well, i.e., for the operator corresponding to our problem.

This operator is symmetric. It remains to check that it is selfadjoint. It is well known that it suffices to prove that for real $s$ with sufficiently large absolute value after adding $i s$ to the potential we have uniqueness for the problem (then there exists a unique solution for all nonzero real $s$ ). Let us look once again at the proof of the a priori estimate. Recalling that the corresponding operators are selfadjoint, instead of (1.4) and (1.5) we can write the inequalities

$$
|s|\left\|\varphi_{1} w\right\|_{0, \Omega} \leq C_{7}\left\|(\mathcal{D}+V+i s)\left(\varphi_{1} w\right)\right\|_{0, \Omega}
$$

and

$$
|s|\left\|\varphi_{2} w\right\|_{0, \Omega} \leq C_{8}\left\|(\mathcal{D}+V+i s)\left(\varphi_{2} w\right)\right\|_{0, \Omega},
$$

where the constants do not depend on $s$. For sufficiently large $|s|$, this obviously yields the estimate

$$
|s|\|w\|_{0, \Omega} \leq C_{9}\|(\mathcal{D}+V+i s) w\|_{0, \Omega},
$$

which implies uniqueness.

The domain $H^{1}(\Omega, B)^{4}$ of the operator $(\mathcal{D}+V)_{\Omega}$ is compactly embedded in $L_{2}(\Omega)^{4}$; therefore, the spectrum of this operator is discrete.

Without loss of generality we may assume that the point $\lambda=0$ does not belong to the spectrum. Then our operator determines a continuous isomorphism between $H^{1}(\Omega, B)^{4}$ and $L_{2}(\Omega)^{4}$. Therefore, the orthonormal basis of eigenfunctions in $L^{2}(\Omega)$ remains to be an unconditional basis in $H^{1}(\Omega, B)^{4}$.

The last assertion of the theorem follows from the theorem on local smoothness of solutions to elliptic problems.

Using interpolation arguments, we conclude that, moreover, for $0<s<1 / 2$ this basis remains to be an unconditional basis in $H^{s}(\Omega)^{4}$, and for $1 / 2<s<1$, in the subspace $H^{s}(\Omega, B)^{4} \subset H^{s}(\Omega)^{4}$ consisting of functions that satisfy the homogeneous boundary condition (7) (cf. [15]).

Note also that, since the operator under consideration acts from $H^{1}(\Omega, B)^{4}$ to $H^{0}(\Omega)^{4}$, we automatically get a lower estimate of proper order for the absolute values of its eigenvalues (see, e.g., [19]).

In conclusion, we note the following. The linear set of smooth functions $w$ on $\bar{\Omega}$ vanishing near the origin and satisfying the boundary condition is dense in $H^{1}(\Omega, B)^{4}$. Therefore, if originally the operator $(\mathcal{D}+V)_{\Omega}$ is defined on such functions, it is essentially selfadjoint. We see that under condition (1.2) the situation in $\Omega$ is similar to that in $\mathbb{R}^{3}$.

\section{$\S 2$. The case of a Coulomb singularity with $|\theta|<1$}

Now we assume that $V(x)$ is a matrix-valued potential of the form (1.1), where $V_{1}(x)$ is smooth in $\bar{\Omega}$ and $V_{0}(x)$ vanishes near $\Gamma$, satisfies the inequality

$$
\left|V_{0}(x)\right| \leq \frac{\theta}{|x|} \quad \text { with } 0<\theta<1,
$$


and is smooth everywhere except the origin; moreover, both matrices are Hermitian. We assume that the potential is of the same form in $\mathbb{R}^{3}$ with finitely supported $V_{j}$. (However, in $\S 3$ we shall see that it is possible to add a constant to the potential, at least not too large, and we shall use this.)

2.1. The Dirac operator with singular potential in $\mathbb{R}^{3}$. The Dirac operator in $\mathbb{R}^{3}$ with a potential that has a Coulomb singularity of type (2.1) was investigated among others by Nenciu [9], Wüst [20]-[22], Klaus-Wüst [23, 24], and Schmincke [25].

In [9] quadratic and sesquilinear forms were used, so the approach is similar to the classical approach to boundary-value problems for strongly elliptic second order systems. The operator $\mathcal{D}$ is not semibounded. Nevertheless, Nenciu showed that the sum of the forms for the operator $\mathcal{D}$ and for the operator of multiplication by the potential determines a selfadjoint operator in $L_{2}$. More precisely, the following statement is true.

Proposition 2.1. There exists a selfadjoint operator $(\mathcal{D}+V)_{\mathbb{R}^{3}}$ in $L_{2}$ such that its domain $H_{V}=H_{V}\left(\mathbb{R}^{3}\right)^{4}$ is a dense subset of $H^{1 / 2}$ and

$$
\left((\mathcal{D}+V)_{\mathbb{R}^{3}} w_{1}, w_{2}\right)_{0, \mathbb{R}^{3}}=\left(\mathcal{D} w_{1}+V w_{1}, w_{2}\right)_{0, \mathbb{R}^{3}} \quad\left(w_{1} \in H_{V}, w_{2} \in H^{1 / 2}\right) .
$$

The operator is determined by these conditions uniquely.

Nenciu used the domain of the operator $|\mathcal{D}|^{1 / 2}$ instead of $H^{1 / 2}$. However,

$$
|\mathcal{D}|^{1 / 2}=\left(\mathcal{D}^{2}\right)^{1 / 4}=(I-\Delta)^{1 / 4} I_{4}
$$

therefore $D\left(|\mathcal{D}|^{1 / 2}\right)$ is simply equal to $H^{1 / 2}$.

Some explanations how Proposition 2.1 was obtained will be given in Subsection 2.3.

Remark 2.2. Since $\mathcal{D}$ is an elliptic first order differential operator with constant coefficients and with nowhere degenerated symbol, it maps $H^{s}$ isomorphically onto $H^{s-1}$ for every $s$. The operator (of multiplication by) $V$ is bounded from $H^{1}$ to $H^{0}$. By duality, it is bounded from $H^{0}$ to $H^{-1}$, and by interpolation arguments it is bounded from $H^{s}$ to $H^{s-1}$ for $0 \leq s \leq 1$.

In particular, $\mathcal{D}$ and $V$ act from $H^{1 / 2}$ to $H^{-1 / 2}$, hence the right-hand side of (2.2) certainly makes sense as a continuous antilinear functional on $H^{1 / 2}$, and the following formula is true:

$$
(\mathcal{D}+V)_{\mathbb{R}^{3}} w_{1}=\mathcal{D} w_{1}+V w_{1}, \quad w_{1} \in H_{V},
$$

as an identity in $H^{-1 / 2}$. However, the domain of the operator $(\mathcal{D}+V)_{\mathbb{R}^{3}}$ consists of $w_{1} \in H^{1 / 2}$ such that the right-hand side of $(2.2)$ is continuous in $w_{2}$ on $H^{1 / 2}$ with respect to the $L_{2}$-norm and the sum on the right-hand side of (2.3) belongs to $L_{2}$.

Obviously, $H^{1 / 2} \subset H_{V} \subset H^{1}$. Under the assumptions of the preceding section, we have $H_{V}=H^{1}$.

For convenience, we mention several local properties of the functions in $H_{V}$.

Proposition 2.3. 1. The function $(\mathcal{D}+V)_{\mathbb{R}^{3}} w, w \in H_{V}$, vanishes outside the support of $w$.

2. Multiplication by any smooth function $\varphi$ is possible in the space $H_{V}$. Moreover, the following "Leibniz formula" is true:

$$
(\mathcal{D}+V)_{\mathbb{R}^{3}}(\varphi w)=\varphi(\mathcal{D}+V)_{\mathbb{R}^{3}} w+\varphi_{1} w, \quad \text { where } \varphi_{1}=\mathcal{D}\left(\varphi I_{4}\right) .
$$

3. If $w \in H_{V}$ and $\varphi$ is a smooth compactly supported function vanishing in a neighborhood of the origin, then $\varphi w \in H^{1}$. In particular, if a compactly supported function $w \in H_{V}$ vanishes in a neighborhood of the origin, then it belongs to $H^{1}$. 
Proof. Statement 1 follows from (2.3).

We check statement 2. Let $w_{1} \in H_{V}$, so that the form $\left(\mathcal{D} w_{1}+V w_{1}, w_{2}\right)_{0}$ is continuous in $w_{2}$ with respect to the $L_{2}$-norm. Then, assuming for simplicity that $\varphi$ is real, we have

$$
\left(\mathcal{D}\left(\varphi w_{1}\right)+V \varphi w_{1}, w_{2}\right)_{0, \mathbb{R}^{3}}=\left(\mathcal{D} w_{1}+V w_{1}, \varphi w_{2}\right)_{0, \mathbb{R}^{3}}+\left(w_{1}, \varphi_{1} w_{2}\right)_{0, \mathbb{R}^{3}}
$$

with obvious meaning for $\varphi_{1}=\mathcal{D}\left(\varphi I_{4}\right)$. Note that the absolute value of the first term on the right-hand side of (2.5) is dominated by $C_{1}\left\|\varphi w_{2}\right\|_{0, \mathbb{R}^{3}}$ and therefore by $C_{2}\left\|w_{2}\right\|_{0, \mathbb{R}^{3}}$. The latter is surely true for the second term as well. Thus, the left-hand side of $(2.5)$ is continuous in $w_{2}$ with respect to the $L_{2}$-norm.

Formula (2.5) implies (2.4).

Now, we check statement 3 . Let $\psi$ be a smooth compactly supported function equal to 1 in a neighborhood of $\operatorname{supp} \varphi$ and vanishing near the origin. By (2.4), from (2.3) we deduce that $(\mathcal{D}+\psi V)(\varphi w) \in L_{2}$. Applying the theorem on local smoothness for solutions of elliptic systems, we conclude that $\varphi w \in H^{1}$.

Nenciu's condition $D\left((\mathcal{D}+V)_{\mathbb{R}^{3}}\right) \subset D\left(|\mathcal{D}|^{1 / 2}\right)$ means that the kinetic energy is finite. In the papers [20]-22] by Wüst, another approach to the construction of a distinguished selfadjoint extension of the operator $\mathcal{D}+V$ was proposed. Namely, the singular potential $V$ was replaced by an approximating family of potentials $V^{(t)}, t \rightarrow \infty$, cut off near the singularity. The Nenciu's condition was replaced by $D\left((\mathcal{D}+V)_{\mathbb{R}^{3}}\right) \subset D\left(|x|^{-1 / 2}\right)$, which means that the potential energy is finite. Further, in the papers [23, 24 by Klaus and Wüst, it was shown that these two extensions coincide and, under the approximation, the resolvents converge in norm. However, in those papers the potential was assumed to be scalar.

2.2. The operator corresponding to the boundary-value problem with a singular potential. Now we consider the boundary-value problem. We denote by $H_{\mathrm{loc}}^{1}(\Omega, B)^{4}$ the space of all functions whose restrictions to $\Omega_{\varepsilon}$ belong to $H^{1}\left(\Omega_{\varepsilon}\right)^{4}$ for an arbitrarily small $\varepsilon>0$ and satisfy the boundary condition $B u=0$.

Using the functions $\varphi_{1}, \varphi_{2}$ and $\psi_{2}$ introduced in the proof of Theorem 1.1, we define the operator corresponding to our problem with singularity as follows:

$$
(\mathcal{D}+V)_{\Omega}=\left.(\mathcal{D}+V)_{\mathbb{R}^{3}}\left(\varphi_{1} \cdot\right)\right|_{\Omega}+\left(\mathcal{D}+\psi_{2} V\right)_{\Omega}\left(\varphi_{2} \cdot\right) .
$$

On the right-hand side, the operator $\left(\mathcal{D}+\psi_{2} V\right)_{\Omega}$ corresponds to an elliptic problem without singularities. This operator acts from $H^{1}(\Omega, B)^{4}$ to $L_{2}(\Omega)^{4}$. The function $\psi_{2}$ may be changed; it must only be equal to 1 in a neighborhood of the support of $\varphi_{2}$ and vanish near the origin.

Denote the domain of the operator $(2.6)$ by $H_{V}(\Omega, B)^{4}$. It consists of functions $w \in$ $L_{2}(\Omega)^{4}$ such that for every function $\varphi \in C_{0}^{\infty}(\Omega)$ supported in a neighborhood of the origin the product $\varphi w$ belongs to $H_{V}\left(\mathbb{R}^{3}\right)^{4}$, and for every $\varphi \in C^{\infty}(\bar{\Omega})$ vanishing in a neighborhood of the origin and equal to 1 outside a larger neighborhood the product $\varphi w$ belongs to $H^{1}(\Omega, B)^{4}$.

Relations (2.6) and (2.3) imply that for $w \in H_{V}(\Omega, B)^{4}$ we have

$$
(\mathcal{D}+V)_{\Omega} w=\mathcal{D} w+V w,
$$

and here the sum on the right-hand side belongs to $H^{0}(\Omega)^{4}$.

We define the norm in the space $H_{V}(\Omega, B)^{4}$ by the formula

$$
\|w\|_{H_{V}(\Omega, B)^{4}}^{2}=\left\|(\mathcal{D}+V)_{\mathbb{R}^{3}}\left(\varphi_{1} w\right)\right\|_{0, \Omega}^{2}+\left\|\varphi_{2} w\right\|_{1, \Omega}^{2}+\|w\|_{0, \Omega}^{2},
$$


where $\varphi_{1}$ and $\varphi_{2}$ are the same functions as above (actually, only the location of their supports and the fact that their sum exceeds a positive constant everywhere are important). Using the theorem on local smoothness for solutions of elliptic problems, we see that the functions in $H_{V}(\Omega, B)^{4}$ belong to the intersection $H^{1 / 2}(\Omega)^{4} \cap H_{\text {loc }}^{1}(\Omega, B)^{4}$.

Remark 2.4. 1. Different choices of the partition of unity and different choices of $\psi_{2}$ produce equivalent definitions of the operator (2.6).

2. The norms corresponding to different partitions of unity are equivalent.

Indeed, first we note that for a smooth function $\varphi$ with support inside $\Omega \backslash\{0\}$ we have

$$
(\mathcal{D}+V)_{\mathbb{R}^{3}}(\varphi \cdot)=(\mathcal{D}+\psi V)_{\Omega}(\varphi \cdot),
$$

where $\psi$ is another smooth function having support inside $\Omega \backslash\{0\}$ and equal to 1 in a neighborhood of $\operatorname{supp} \varphi$.

Now let functions $\widetilde{\varphi}_{j}, j=1,2$, form another partition of unity with the same properties as $\varphi_{j}$, and let $\widetilde{\psi}_{2}$ be a function smooth in $\bar{\Omega}$, equal to 1 in a neighborhood of the support of $\widetilde{\varphi}_{2}$, and vanishing near the origin. We compare two definitions, with $\varphi_{j}, \psi_{2}$ and with $\widetilde{\varphi}_{j}, \widetilde{\psi}_{2}$. Without loss of generality we assume that $\psi_{2}=\widetilde{\psi}_{2}$. Taking Proposition 2.3 into account, we can write the definition (2.6) with the functions $\varphi_{j}$ as

$$
(\mathcal{D}+V)_{\Omega} w=\left.(\mathcal{D}+V)_{\mathbb{R}^{3}}\left(\left(\widetilde{\varphi}_{1}+\widetilde{\varphi}_{2}\right) \varphi_{1} w\right)\right|_{\Omega}+\left(\mathcal{D}+\psi_{2} V\right)_{\Omega}\left(\left(\widetilde{\varphi}_{1}+\widetilde{\varphi}_{2}\right) \varphi_{2} w\right) .
$$

By (2.9), we have

$$
\left.(\mathcal{D}+V)_{\mathbb{R}^{3}}\left(\widetilde{\varphi}_{2} \varphi_{1} w\right)\right|_{\Omega}=(\mathcal{D}+V)_{\Omega}\left(\widetilde{\varphi}_{2} \varphi_{1} w\right)
$$

Therefore,

$$
(\mathcal{D}+V)_{\Omega} w=\left.(\mathcal{D}+V)_{\mathbb{R}^{3}}\left(\widetilde{\varphi}_{1} \varphi_{1} w\right)\right|_{\Omega}+\sum_{\{i, j\} \neq\{1,1\}}\left(\mathcal{D}+\psi_{2} V\right)_{\Omega}\left(\widetilde{\varphi}_{i} \varphi_{j} w\right) .
$$

The same expression is obtained if we start with the functions $\widetilde{\varphi}_{j}$.

Now we outline how to estimate the norm in (2.8) via the analogous norm with $\widetilde{\varphi}_{j}$. For example, we have

$$
\begin{aligned}
& \left\|(\mathcal{D}+V)_{\mathbb{R}^{3}}\left(\varphi_{1} w\right)\right\|_{0, \Omega} \\
& \quad \leq\left\|(\mathcal{D}+V)_{\mathbb{R}^{3}}\left(\widetilde{\varphi}_{1} \varphi_{1} w\right)\right\|_{0, \Omega}+\left\|(\mathcal{D}+V)\left(\widetilde{\varphi}_{2} \varphi_{1} w\right)\right\|_{0, \Omega} \\
& \quad \leq\left\|\varphi_{1}(\mathcal{D}+V)_{\mathbb{R}^{3}}\left(\widetilde{\varphi}_{1} w\right)\right\|_{0, \Omega}+\left\|\left(\mathcal{D} \varphi_{1}\right)\left(\widetilde{\varphi}_{1} w\right)\right\|_{0, \Omega}+C_{1}\left\|\widetilde{\varphi}_{2} \varphi_{1} w\right\|_{1, \Omega} \\
& \quad \leq C_{2}\left[\left\|(\mathcal{D}+V)_{\mathbb{R}^{3}}\left(\widetilde{\varphi}_{1} w\right)\right\|_{0, \Omega}+\left\|\widetilde{\varphi}_{2} w\right\|_{1, \Omega}+\|w\|_{0, \Omega}\right] .
\end{aligned}
$$

And so on.

The space $H_{V}(\Omega, B)^{4}$ is complete and therefore is a Hilbert space. (The inner product corresponding to the norm (2.8) can be written in an obvious way.)

This assertion follows from the completeness of the space $H_{V}\left(\mathbb{R}^{3}\right)^{4}$ endowed with the graph norm for the operator $(\mathcal{D}+V)_{\mathbb{R}^{3}}$.

In contrast with the situation in the preceding section, we cannot consider our problem in usual Sobolev spaces. Instead, we have endowed the domain of the operator with its graph norm, and we can use the fact that the operator in $\mathbb{R}^{3}$ and the operator without singularity in $\Omega$ are selfadjoint. This yields the required result.

Theorem 2.5. The operator (2.6) with domain $H_{V}(\Omega, B)^{4}$ is selfadjoint in $L_{2}(\Omega)^{4}$. Its spectrum is discrete, and there exists a basis of its eigenfunctions orthonormal in $L_{2}(\Omega)^{4}$. The latter remains to be an unconditional basis in $H_{V}(\Omega, B)^{4}$.

If $V(x)$ for $x \neq 0$ and the boundary $\Gamma$ are infinitely smooth, then the eigenfunctions belong to $C^{\infty}(\bar{\Omega} \backslash\{0\})$. 
Proof. It is not difficult to check that $(\mathcal{D}+V)_{\Omega}$ is a symmetric operator. Indeed, multiplying (2.6) by $\varphi_{1}+\varphi_{2}$, we obtain the sum of four operators

$$
\begin{aligned}
\varphi_{1}(\mathcal{D} & +V)_{\mathbb{R}^{3}}\left(\varphi_{1} \cdot\right)+\varphi_{2}(\mathcal{D}+V)_{\mathbb{R}^{3}}\left(\varphi_{1} \cdot\right) \\
& +\varphi_{1}\left(\mathcal{D}+\psi_{2} V\right)_{\Omega}\left(\varphi_{2} \cdot\right)+\varphi_{2}\left(\mathcal{D}+\psi_{2} V\right)_{\Omega}\left(\varphi_{2} \cdot\right),
\end{aligned}
$$

where in the first two terms extension by zero outside $\Omega$ and restriction to $\Omega$ are assumed. The 1st and 4th terms are selfadjoint operators because the operator $(\mathcal{D}+V)_{\mathbb{R}^{3}}$ in $L_{2}\left(\mathbb{R}^{3}\right)^{4}$ and the operator $\left(\mathcal{D}+\psi_{2} V\right)_{\Omega}$ in $L_{2}(\Omega)^{4}$ are selfadjoint. Now we verify that the 2 nd and 3rd terms are formally adjoint to each other on functions in $H^{1}(\Omega)^{4}$. We consider the integral

$$
\int_{\Omega} \varphi_{2}(\mathcal{D}+V)_{\mathbb{R}^{3}}\left(\varphi_{1} w_{1}\right) \cdot \bar{w}_{2} d x
$$

Since the integrand vanishes outside $\operatorname{supp} \varphi_{1}$, we can insert the factor $\psi_{1}$ before $\bar{w}_{2}$. After this we can extend our integral over $\mathbb{R}^{3}$. Since the operator $(\mathcal{D}+V)_{\mathbb{R}^{3}}$ is selfadjoint, we get

$$
\int_{\mathbb{R}^{3}} \varphi_{1} w_{1} \cdot \overline{(\mathcal{D}+V)_{\mathbb{R}^{3}}\left(\psi_{1} \varphi_{2} w_{2}\right)} d x
$$

Since supp $\psi_{1} \varphi_{2} \subset \Omega \backslash\{0\}$, we can replace $(\mathcal{D}+V)_{\mathbb{R}^{3}}$ by $\left(\mathcal{D}+\psi_{2} V\right)_{\Omega}$ and integrate again over $\Omega$. Furthermore, the function $\mathcal{D} \psi_{1}$ vanishes on $\operatorname{supp} \varphi_{1}$, and hence $\psi_{1}$ can be taken out of the operator sign in $\left(\mathcal{D}+\psi_{2} V\right)_{\Omega}$ and dropped thereafter. Thus, we get

$$
\int_{\Omega} w_{1} \cdot \overline{\varphi_{1}\left(\mathcal{D}+\psi_{2} V\right)_{\Omega}\left(\varphi_{2} w_{2}\right)} d x
$$

as required; here the 3rd operator in (2.10) acts on $w_{2}$.

Now, we check that $(\mathcal{D}+V)_{\Omega}$ is a bounded Fredholm operator from $H_{V}(\Omega, B)^{4}$ to $L_{2}(\Omega)^{4}$ with index zero.

We can verify this in the same way as in the preceding section. First, we note that, by the definition (2.8) of the norm in $H_{V}(\Omega, B)^{4}$, our operator is automatically bounded from $H_{V}(\Omega, B)^{4}$ to $L_{2}(\Omega)^{4}$.

Now we check the a priori estimate

$$
\|u\|_{H_{V}(\Omega, B)^{4}} \leq C_{3}\left[\left\|(\mathcal{D}+V)_{\Omega} u\right\|_{0, \Omega}+\|u\|_{0, \Omega}\right] .
$$

Namely, let $w \in H_{V}(\Omega, B)^{4}$, and let $s_{0}$ be a nonzero real number. Then

$$
\left\|\varphi_{1} w\right\|_{H_{V}(\Omega, B)^{4}} \leq C_{4}\left\|\left(\mathcal{D}+V+i s_{0}\right)_{\mathbb{R}^{3}}\left(\varphi_{1} w\right)\right\|_{0, \Omega}
$$

and

$$
\left\|\varphi_{2} w\right\|_{1, \Omega} \leq C_{5}\left\|\left(\mathcal{D}+\psi_{2} V+i s_{0}\right)_{\Omega}\left(\varphi_{2} w\right)\right\|_{0, \Omega},
$$

because the corresponding operators with $s_{0}=0$ are selfadjoint, respectively, in $L_{2}^{4}$ and $L_{2}(\Omega)^{4}$. This implies (2.11).

Furthermore, these operators with real $s_{0} \neq 0$ have inverses $R_{1}$ and $R_{2}$. A right parametrix is constructed as in (1.7).

Thus, $(\mathcal{D}+V)_{\Omega}$ is a Fredholm operator. Replacing $V$ by $t V, 0 \leq t \leq 1$, we see that $(\mathcal{D}+V)_{\Omega}$ has index zero.

For a real $s_{0}$ with sufficiently large absolute value, we check that $\left(\mathcal{D}+V+i s_{0}\right)_{\Omega}$ is an invertible operator. Its spectrum is discrete because the inverse operator maps $L_{2}(\Omega)^{4}$ to $H^{1 / 2}(\Omega)^{4}$. Without loss in generality we may assume that invertibility takes place for $s_{0}=0$, and hence we see that the eigenfunctions form an unconditional basis in the operator's domain.

\footnotetext{
${ }^{2}$ In places like this we write the inner product of vectors $w_{1}$ and $w_{2}$ with complex components in the form $w_{1} \cdot \bar{w}_{2}$.
} 
2.3. Nenciu's abstract approach. Thus, the operator $(\mathcal{D}+V)_{\Omega}$ is constructed and Theorem 2.5 is proved. Now we briefly discuss the abstract approach proposed in 9 . As has already been mentioned, it was used in [9] for the investigation of the Dirac operator with singular potential in $\mathbb{R}^{3}$, and the result cited above was obtained in [9] (see Proposition 2.1). In the next section we show that Nenciu's approach enables us to define the operator $(\mathcal{D}+V)_{\Omega}$ in an alternative way. This approach involves an important formula for the resolvent of the operator under investigation (see (2.21) below).

Let $H$ be a Hilbert space with inner product $(\cdot, \cdot)$, and let $A$ and $V$ be two selfadjoint operators in $H$ with domains $D(A)$ and $D(V)$, respectively. Assum 3 that the operator $|A|$ is positive definite, $D(V) \supset D(A)$, and the inequality

$$
\||V| f\| \leq C\||A| f\| \quad(f \in D(|A|))
$$

is true (of course, $D(|A|)$ coincides with $D(A)$ ). Then by the Heinz theorem (see, e.g., 26 $\S 12]) D\left(|V|^{1 / 2}\right) \supset D\left(|A|^{1 / 2}\right)$ and

$$
\left\||V|^{1 / 2} f\right\| \leq C\left\||A|^{1 / 2} f\right\| \quad\left(f \in D\left(|A|^{1 / 2}\right)\right) .
$$

We write the polar decompositions of $A$ and $V$ in the form $A=T|A|, V=S|V|$, where $T f=f$ if $A f=0$, and $S f=f$ if $V f=0$. The sesquilinear forms of the operators $A$ and $V$ are

$$
\begin{aligned}
& h_{A}[f, g]=\left(|A|^{1 / 2} f, T|A|^{1 / 2} g\right), \\
& h_{V}[f, g]=\left(|V|^{1 / 2} f, S|V|^{1 / 2} g\right) .
\end{aligned}
$$

On $D\left(|A|^{1 / 2}\right)$ both of them are defined, and by (2.13) we have

$$
\left|h_{V}[f, f]\right| \leq C\left\||A|^{1 / 2} f\right\|^{2} \quad\left(f \in D\left(|A|^{1 / 2}\right) .\right.
$$

Let $W$ be a bounded operator in $H$ such that $W|A|^{-1 / 2}$ is a compact operator. Now we formulate some conditions that guarantee the existence of a unique operator $B$ with domain $D(B) \subset D\left(|A|^{1 / 2}\right)$ such that

$$
(B f, g)=h_{A}[f, g]+h_{V}[f, g]+h_{W}[f, g] \quad\left(f \in D(B), g \in D\left(|A|^{1 / 2}\right)\right) .
$$

To do this, we assume that $z \notin \sigma(A)$ (i.e., $z$ does not belong to the spectrum of $A$ ) and consider the operator

$$
M(z)=|V|^{1 / 2}(A-z)^{-1}|V|^{1 / 2} .
$$

Initially, this operator is defined on $D\left(|V|^{1 / 2}\right)$, but it is bounded and admits a bounded extension to $H$ by the formula

$$
M(z)=\left\{|V|^{1 / 2}|A|^{-1 / 2}\right\}\left\{|A|(A-z)^{-1}\right\}\left\{\left(|V|^{1 / 2}|A|^{-1 / 2}\right)^{*}\right\} .
$$

We also see that $M(z)$ depends on $z$ analytically. Assume that

$$
\left\|M\left(z_{0}\right)\right\|<1 \quad \text { for some } z_{0} \notin \sigma(A),
$$

so that $I+S M(z)$ is invertible for $z=z_{0}$. Furthermore, assume that the operator $|V|^{1 / 2}\left(A-z_{0}\right)^{-1}(A-z)^{-1}|V|^{1 / 2}$ extends by continuity to a compact operator for $z \notin \sigma(A)$.

Proposition 2.6. Under the conditions listed above (including (2.12), (2.19), and (2.20)), there exists a unique selfadjoint operator $B$ with domain $D(B) \subset D(A)$ such that $(2.16)$ is true.

\footnotetext{
${ }^{3}$ To simplify the presentation, we restrict generality, as compared with 9 .
} 
This assertion is contained in [9, Corollary 2.1]. In 9] that corollary was applied in $\mathbb{R}^{3}$ to the free Dirac operator as $A$, multiplication by the singular part of the potential as $V$, multiplication by its smooth part as $W$, and the full Dirac operator with the potential consisting of the singular and smooth parts as $B$. In the next section we shall show that our operator $(\mathcal{D}+V)_{\Omega}$ can be obtained in a similar way if for $A$ we take either the operator $(\mathcal{D})_{\Omega}$ corresponding to the free Dirac system in $\Omega$ (if this operator has bounded inverse) or, otherwise, the operator $(\mathcal{D}+c)_{\Omega}$ with a small real constant $c$ added to gain invertibility. The proof will be based on specific results established in [9] for the Dirac operator in $\mathbb{R}^{3}$.

The following results of [9] will be particularly important.

Proposition 2.7. 1. Under the same assumptions, the operator $(I+S M(z))^{-1}$ is a meromorphic function of $z$ outside $\sigma(A)$, and hence it exists in $\mathbb{C} \backslash \sigma(A)$ at least outside a set without limit points in $\mathbb{C} \backslash \sigma(A)$.

2. If $z \notin \sigma(A)$ and the operator $I+S M(z)$ is invertible, then $z \notin \sigma(B)$ and the following formula for the difference of the resolvents for $B$ and $A$ is true:

$$
\begin{aligned}
& (B-z)^{-1}-(A-z)^{-1} \\
& \quad=-\left(|V|^{-1 / 2}(A-\bar{z})^{-1}\right)^{*}(I+S M(z))^{-1} S|V|^{1 / 2}(A-z)^{-1} .
\end{aligned}
$$

Since the spectrum of our operator $A=(\mathcal{D}+V)_{\Omega}$ is discrete, we can use this formula for almost all real $z$.

\section{§3. The asymptotic Behavior of Eigenvalues}

Here our main goal is to prove the following theorem.

Theorem 3.1. Under the assumptions of $\S 2$, the following formula is valid for the eigenvalues of the spectral problem I:

$$
\lambda_{n}=b n^{1 / 3}+o\left(|n|^{1 / 3}\right)(n \rightarrow \pm \infty), \quad \text { where } b=\left(\frac{3 \pi^{2}}{\operatorname{meas} \Omega}\right)^{1 / 3} .
$$

The key point of the proof is the use of a formula of type (2.21) for the difference of the resolvents of the operators corresponding to our problem with a singular potential and without it. First, in Subsections 3.1-3.4 we check that our operator $(\mathcal{D}+V)_{\Omega}$ can also be obtained by the Nenciu approach (see Subsection 2.3) and satisfies the assumptions that ensure the above formula.

3.1. Notation. In this section it will be convenient to change our notation slightly. Consider the problem

$$
(\mathcal{D}+c) w=f(x \in \Omega), \quad B w=0 \quad(x \in \Gamma) .
$$

If uniqueness holds for $c=0$, then we put here $c=0$; otherwise we fix a real $c$ with a small absolute value for which we have uniqueness. Below, we shall see how small it should be; in particular, we assume that $|c|<1$. By $L_{0}$ we denote the corresponding operator in $L_{2}(\Omega)^{4}$. We shall write $\mathcal{D}_{c}$ instead of $\mathcal{D}+c$. The same notation will be used for the corresponding operator in $L_{2}\left(\mathbb{R}^{3}\right)^{4}$. The potential $V$ will be added to $\mathcal{D}_{c}$. This potential will be assumed to satisfy inequality (2.1) with $\theta<1$, to vanish near the boundary, and to be extended by zero outside $\Omega$. This does not restrict the generality of the proof, because a smooth term in $\bar{\Omega}$ has no influence on the asymptotics. In general the total potential is not compactly supported, but is equal to the constant $c$ near infinity. We denote by $L$ the operator in $L_{2}(\Omega)^{4}$ that corresponds to the problem under investigation:

$$
\left(\mathcal{D}_{c}+V\right) w=f(x \in \Omega), \quad B w=0(x \in \Gamma) .
$$


For functions defined on $\mathbb{R}^{3}$, we denote by $P$ the operator of restriction to $\Omega$; next, by $P^{*}$ we denote the operator of extension of functions defined on $\Omega$ to the entire $\mathbb{R}^{3}$ by zero outside $\Omega$ (above, these operators were used implicitly). Let $V=S|V|$ be the polar decomposition of the matrix $V$.

3.2. The domain of the operator $\left|L_{0}\right|^{1 / 2}$ and the boundedness of the form of $V$ on it. By the Hardy inequality (8) and the a priori estimate for the uniquely solvable elliptic problem (3.2), we have

$$
\|V w\|_{0, \Omega} \leq C_{1}\left\|L_{0} w\right\|_{0, \Omega}
$$

for $w$ in $H^{1}(\Omega, B)^{4}$. This inequality remains true if we replace $V$ by $|V|$ and $L_{0}$ by $\left|L_{0}\right|$ (cf. (2.12)).

Denote by $H_{1 / 2}=H^{1 / 2}(\Omega, B)^{4}$ the domain of the operator $\left|L_{0}\right|^{1 / 2}$. The standard description of such a domain is as follows: if $\left\{w_{j}\right\}_{1}^{\infty}$ is the orthonormal basis in $L_{2}(\Omega)^{4}$ consisting of the eigenfunctions of $L_{0}$, and $\lambda_{j}$ are the corresponding eigenvalues, then a function $w$ in $L_{2}(\Omega)^{4}, w=\sum c_{j}(w) w_{j}$, belongs to $H_{1 / 2}$ if and only if the norm defined by the formula

$$
\|w\|_{1 / 2}^{2}=\left\|\left|L_{0}\right|^{1 / 2} w\right\|_{0, \Omega}^{2}=\sum\left|\lambda_{j} \| c_{j}(w)\right|^{2}
$$

is finite. Below we shall see that $H_{1 / 2}$ is a linear subset of the Sobolev space $H^{1 / 2}(\Omega)^{4}$, and we describe this subset.

By the Heinz theorem, the operator $|V|^{1 / 2}$ is defined on $H^{1 / 2}$ and

$$
\left\||V|^{1 / 2} w\right\|_{0, \Omega} \leq C_{2}\|w\|_{1 / 2}
$$

(cf. (2.13)). On the same space, the sesquilinear form of the operator $V$ is defined:

$$
h_{V}\left[w_{1}, w_{2}\right]=\left(|V|^{1 / 2} w_{1}, S|V|^{1 / 2} w_{2}\right)_{0, \Omega},
$$

and we see that it is bounded on this space:

$$
\left|h_{V}[w, w]\right| \leq C_{3}\|w\|_{1 / 2}^{2}
$$

(cf. $(2.15))$.

We return to the space $H_{1 / 2}$. It is contained in the Hilbert scale of the domains $H_{\vartheta}$ of the operators $\left|L_{0}\right|^{\vartheta}, 0 \leq \vartheta \leq 1$. This scale coincides with the interpolation scale both for the real and the complex interpolation methods up to norm equivalence (see [27] Subsection 1.18.10, Remark 34). The real and complex interpolation methods for the Sobolev spaces determined by boundary conditions were investigated in the papers by Grisvard [28] and Seeley [29], respectively. Their results imply that $H_{1 / 2}$ is a linear subset of the space $H^{1 / 2}(\Omega)^{4}$ determined by the boundary condition $B u=0$ in the following sense. 1) Let $\rho(x)$ be a positive continuous function on $\bar{\Omega}$ such that near the boundary $\Gamma$ it coincides with the distance from the point $x$ to the boundary. Then the function $\rho^{-1 / 2}(x) B w(x)$ is in $L_{2}(\Omega)^{4}$. 2) The extension of $B w(x)$ by zero outside $\Omega$ belongs to $H^{1 / 2}\left(\mathbb{R}^{3}\right)^{4}$. Here by $B$ we denote a reasonable continuation of the operator (7). The equivalence of these two conditions was verified directly in [30, Section 3.6].

However, it should be mentioned that, actually, these details of understanding the boundary condition are inessential for us, because the functions in the domain of our operator are in $H^{1}\left(\Omega_{\varepsilon}, B\right)^{4}$ for every $\varepsilon>0$ and therefore the boundary condition can be treated in the usual sense of traces of functions in $H^{1}(\Omega)$.

\footnotetext{
${ }^{4}$ The authors are obliged to V. I. Ovchinnikov for this reference.
} 
3.3. The operator $M(z)$ and the invertibility of $I+S M(z)$ for $z=i s$ with large $s$. Recall that $L_{0}$ is a selfadjoint operator with discrete spectrum. We define the operator $M(z)$ outside this spectrum by the formula

$$
M(z)=|V|^{1 / 2}\left(L_{0}-z\right)^{-1}|V|^{1 / 2}
$$

(cf. (2.17)). A priori, it is defined on $H^{1 / 2}(\Omega)^{4}$, but it extends to a bounded operator on $H^{0}(\Omega)^{4}$ by the formula

$$
M(z)=\left\{|V|^{1 / 2}\left|L_{0}\right|^{-1 / 2}\right\}\left\{\left|L_{0}\right|\left(L_{0}-z\right)^{-1}\right\}\left\{\left(|V|^{1 / 2}\left|L_{0}\right|^{-1 / 2}\right)^{*}\right\}
$$

(cf. (2.18)).

Proposition 3.2. The norm of $M(z)$ is less than 1 for $z=i s$ with real s having suffciently large absolute value. Thus, the operator $I+S M(z)$ is invertible for such $z$.

This assertion will be deduced from a similar one established in [9] for operators in $\mathbb{R}^{3}$. For this reason, we shall need the following auxiliary operators:

$$
\begin{aligned}
& M_{1}(z)=|V|^{1 / 2} P\left(\mathcal{D}_{c}-z\right)^{-1} P^{*}|V|^{1 / 2}, \\
& M_{2}(z)=|V|^{1 / 2}\left(\mathcal{D}_{c}-z\right)^{-1}|V|^{1 / 2}, \\
& M_{3}(z)=|V|^{1 / 2}(\mathcal{D}-z)^{-1}|V|^{1 / 2} .
\end{aligned}
$$

The first of these operators is defined on functions on $\Omega$ that lie in the domain of $|V|^{1 / 2}$; the second and the third operators are defined on functions on $\mathbb{R}^{3}$ that lie in the domain of essentially the same operator. But we shall see soon that $M_{1}(z)$ extends to a bounded operator in $L_{2}(\Omega)^{4}$, and $M_{2}(z)$ and $M_{3}(z)$ extend to bounded operators in $L_{2}\left(\mathbb{R}^{3}\right)^{4}$. As functions of $z$, the operators $M_{1}(z)$ and $M_{2}(z)$ are defined for $z$ outside the spectrum $\sigma\left(\mathcal{D}_{c}\right)$, and $M_{3}(z)$ is defined outside $\sigma(\mathcal{D})$, in particular, for nonreal $z$. However, all of them are defined on some interval of the real axis as well, because the spectrum of $\mathcal{D}$ contains no points of the interval $(-1,1)$ (see [7, Subsection 1.4.4]) and $c$ is assumed to be in this interval.

To see that $M_{2}(z)$ and $M_{3}(z)$ extend to bounded operators in $L_{2}\left(\mathbb{R}^{3}\right)^{4}$, we may refer to Remark 2.2. In the definition of $M_{1}(z)$, the operators $P$ and $P^{*}$ can be commuted with $|V|^{1 / 2}$, and we see that

$$
\left\|M_{1}(z)\right\| \leq\left\|M_{2}(z)\right\| \text {. }
$$

From [9 Section 5] it follows that under our assumptions about the potential we have

$$
\left\|M_{3}(i s)\right\| \leq b_{1}<1, \quad s \in \mathbb{R}
$$

(see also [24]). Now we check that the same is true for $M_{2}(i s)$ if $c$ is sufficiently small. To do this, we consider the difference

$$
\begin{aligned}
& M_{3}(i s)-M_{2}(i s) \\
& \quad=|V|^{1 / 2}\left[(\mathcal{D}-i s)^{-1}-(\mathcal{D}+c-i s)^{-1}\right]|V|^{1 / 2} \\
& \quad=c\left\{|V|^{1 / 2}|\mathcal{D}|^{-1 / 2}\right\}\left\{|\mathcal{D}|(\mathcal{D}-i s)^{-1}\right\}\left\{(\mathcal{D}+c-i s)^{-1}\right\}\left\{\left(|V|^{1 / 2}|\mathcal{D}|^{-1 / 2}\right)^{*}\right\} \\
& \quad=T_{1} T_{2}(s) T_{3}(c, s) T_{4} .
\end{aligned}
$$

Here the operators $T_{1}$ and $T_{4}$ are bounded. Using the spectral theorem, we can check that the operator $T_{2}(s)$ is bounded uniformly in $s$ and $T_{3}(c, s)$ is bounded uniformly in $s$ and $c,|c| \leq 1 / 2$ (these operators are functions of the selfadjoint operator $\mathcal{D}$ bounded on its spectrum). (The same can be checked by passing to Fourier transforms.) Thus, the norm of the operator (3.14) tends uniformly to zero as $c \rightarrow 0$. 
Now, we assume that $c$ is chosen in such a way that

$$
\left\|M_{2}(i s)\right\| \leq b_{2}<1
$$

say, with the constant $b_{2}=\left(1+b_{1}\right) / 2$.

To prove Proposition 3.2, it remains to verify the following statement.

Proposition 3.3. The norm of the operator $M(z)-M_{1}(z)$ in $H^{0}(\Omega)^{4}$ tends to zero as $z=i s, s \rightarrow \pm \infty$.

Proof. Let $f_{1}, f_{2} \in L_{2}(\Omega)^{4}=H^{0}(\Omega)^{4}$. Consider the sesquilinear form of the operator $\left(L_{0}-z\right)^{-1}-P\left(\mathcal{D}_{c}-z\right)^{-1} P^{*}$. It can be written as

$$
h\left(f_{1}, f_{2}\right)=\left(f_{1},\left(L_{0}-\bar{z}\right)^{-1} f_{2}\right)_{0, \Omega}-\left(\left(\mathcal{D}_{c}-z\right)^{-1} P^{*} f_{1}, P^{*} f_{2}\right)_{0, \mathbb{R}^{3}} .
$$

We set

$$
w_{1}=\left(\mathcal{D}_{c}-z\right)^{-1} P^{*} f_{1}, \quad w_{2}=\left(L_{0}-\bar{z}\right)^{-1} f_{2} .
$$

Now the form (3.16) turns into

$$
\left(P\left(\mathcal{D}_{c}-z\right) w_{1}, w_{2}\right)_{0, \Omega}-\left(P w_{1},\left(\mathcal{D}_{c}-\bar{z}\right) w_{2}\right)_{0, \Omega},
$$

where $\mathcal{D}_{c}$ is viewed (only in this formula) as a differential operator in $\Omega$. Integrating by parts, we obtain

$$
h\left(f_{1}, f_{2}\right)=\left(\Phi \gamma w_{1}, \gamma w_{2}\right)_{0, \Gamma},
$$

where $\gamma$ is the operator of restriction to the boundary and $\Phi$ is some smooth matrix on $\Gamma$. Using (3.17), we rewrite (3.19) in the form

$$
\begin{aligned}
h\left(f_{1}, f_{2}\right) & =\left(\Phi \gamma P\left(\mathcal{D}_{c}-z\right)^{-1} P^{*} f_{1}, \gamma\left(L_{0}-\bar{z}\right)^{-1} f_{2}\right)_{0, \Gamma} \\
& =\left(\left(\gamma\left(L_{0}-\bar{z}\right)^{-1}\right)^{*} \Phi \gamma P\left(\mathcal{D}_{c}-z\right)^{-1} P^{*} f_{1}, f_{2}\right)_{0, \Omega} .
\end{aligned}
$$

Here $\gamma\left(L_{0}-\bar{z}\right)^{-1}$ is a bounded operator from $H^{0}(\Omega)^{4}$ to $H^{1 / 2}(\Gamma)^{4}$ and consequently to $H^{0}(\Gamma)^{4}$. The adjoint $\left(\gamma\left(L_{0}-\bar{z}\right)^{-1}\right)^{*}$ is bounded at least as an operator from $H^{0}(\Gamma)^{4}$ to $H^{0}(\Omega)^{4}$.

Therefore,

$$
\left(L_{0}-z\right)^{-1}-P\left(\mathcal{D}_{c}-z\right)^{-1} P^{*}=\left(\gamma\left(L_{0}-\bar{z}\right)^{-1}\right)^{*} \Phi \gamma P\left(\mathcal{D}_{c}-z\right)^{-1} P^{*} .
$$

Such a way of representing the difference of the resolvents of two operators with different domains was proposed by M. Sh. Birman in [31]. Relations (3.9), (3.11), and (3.21) yield

$$
M(z)-M_{1}(z)=T_{1}(z) T_{2}(z),
$$

where

$$
\begin{aligned}
& T_{1}(z)=|V|^{1 / 2}\left(\gamma\left(L_{0}-\bar{z}\right)^{-1}\right)^{*}, \\
& T_{2}(z)=\Phi \gamma P\left(\mathcal{D}_{c}-z\right)^{-1} P^{*}|V|^{1 / 2} .
\end{aligned}
$$

The second of these two operators transforms functions on $\Omega$ to functions on $\Gamma$, and the first transforms functions on $\Gamma$ to functions on $\Omega$.

Now, we verify that $T_{1}(z)$ can be extended to a bounded operator from $H^{0}(\Omega)^{4}$ to $H^{0}(\Gamma)^{4}$ for $z=i s$ with real $s$, with a bound independent of $s$. Let $\psi$ be a scalar function in $C_{0}^{\infty}(\Omega)$ such that $\psi V=V$. Then $(\psi-1) V=0,(\psi-1)|V|^{1 / 2}=0$, thus $\psi|V|^{1 / 2}=|V|^{1 / 2}$.

For functions $g$ on $\Gamma$ and $f$ on $\Omega$, we have

$$
\begin{aligned}
& \left(\psi\left(\gamma\left(L_{0}-\bar{z}\right)^{-1}\right)^{*} g, f\right)_{0, \Omega} \\
& \quad=\left(g, \gamma\left(L_{0}-\bar{z}\right)^{-1} \psi f\right)_{0, \Gamma}=\left(\left(\gamma\left(L_{0}-\bar{z}\right)^{-1} \psi \cdot\right)^{*} g, f\right)_{0, \Omega}
\end{aligned}
$$


hence

$$
T_{1}(z)=|V|^{1 / 2}\left(\gamma\left(L_{0}-\bar{z}\right)^{-1} \psi \cdot\right)^{*} .
$$

Here we commute the operator $\left(L_{0}-\bar{z}\right)^{-1}$ and the operator of multiplication by $\psi$, and then use the identity $\gamma \psi \cdot=0$. This leads to the formula

$$
T_{1}(z)=|V|^{1 / 2}\left(\gamma\left(L_{0}-\bar{z}\right)^{-1}[\psi, \mathcal{D}]^{*}\left(L_{0}-\bar{z}\right)^{-1}\right)^{*} .
$$

Here we have

$$
\begin{aligned}
& \left(\left(\gamma\left(L_{0}-\bar{z}\right)^{-1}[\psi, \mathcal{D}]\left(L_{0}-\bar{z}\right)^{-1}\right)^{*} g, f\right)_{0, \Omega} \\
& \quad=\left(g, \gamma\left(L_{0}-\bar{z}\right)^{-1}[\psi, \mathcal{D}]\left(L_{0}-\bar{z}\right)^{-1} f\right)_{0, \Gamma} \\
& \quad=\left(\left(\gamma\left(L_{0}-\bar{z}\right)^{-1}\right)^{*} g,[\psi, \mathcal{D}]\left(L_{0}-\bar{z}\right)^{-1} f\right)_{0, \Omega} \\
& \left.\quad=\left(\left(L_{0}-z\right)^{-1}[\psi, \mathcal{D}]^{*}\left(\gamma\left(L_{0}-\bar{z}\right)\right)^{-1}\right)^{*} g, f\right)_{0, \Omega}
\end{aligned}
$$

Hence

$$
T_{1}(z)=\left\{|V|^{1 / 2}\left(L_{0}-z\right)^{-1}\right\}\left\{[\psi, \mathcal{D}]^{*}\right\}\left\{\left(\gamma\left(L_{0}-\bar{z}\right)^{-1}\right)^{*} g\right\} .
$$

Here the middle factor is a smooth function not depending on $z$. The left factor can be rewritten in the form

$$
\left\{|V|^{1 / 2}\left|L_{0}\right|^{-1 / 2}\right\}\left\{\left|L_{0}\right|^{1 / 2}\left(L_{0}-i s\right)^{-1}\right\} .
$$

Clearly, this is an operator bounded uniformly in $s$. Finally,

$$
\gamma\left(L_{0}-\bar{z}\right)^{-1}=\left\{\gamma L_{0}^{-1}\right\}\left\{L_{0}\left(L_{0}+i s\right)^{-1}\right\},
$$

and we see that this is a uniformly bounded operator from $H^{0}(\Omega)^{4}$ to $H^{0}(\Gamma)^{4}$, so that the adjoint operator from $H^{0}(\Gamma)^{4}$ to $H^{0}(\Omega)^{4}$ is uniformly bounded as well.

Now we verify that $T_{2}(z)$ can be extended to a bounded operator from $H^{0}(\Omega)^{4}$ to $H^{0}(\Gamma)^{4}$ and that its norm tends to zero as $z=i s, s \rightarrow \pm \infty$. Since $\gamma \psi \cdot=0$, with the same function $\psi$ we have

$$
T_{2}(z)=\Phi \gamma P\left[\left(\mathcal{D}_{c}-z\right)^{-1}, \psi\right] P^{*}|V|^{1 / 2} .
$$

We rewrite this formula as

$$
\begin{aligned}
T_{2}(z)= & \Phi \gamma P\left(\mathcal{D}_{c}-z\right)^{-1}[\psi, \mathcal{D}]\left(\mathcal{D}_{c}-z\right)^{-1} P^{*}|V|^{1 / 2} \\
= & \left\{\Phi \gamma P|\mathcal{D}|^{-1 / 2-\varepsilon}\right\}\left\{|\mathcal{D}|^{1 / 2+\varepsilon}\left(\mathcal{D}_{c}-z\right)^{-1}\right\}\{[\psi, \mathcal{D}]\} \\
& \times\left\{\left(\mathcal{D}_{c}-z\right)^{-1}|\mathcal{D}|^{1 / 2}\right\}\left\{\left(|V|^{1 / 2}|\mathcal{D}|^{-1 / 2}\right)^{*} P^{*}\right\}
\end{aligned}
$$

with $\varepsilon \in(0,1 / 2)$. Here the fifth factor is a bounded operator from $H^{0}(\Omega)^{4}$ to $H^{0}\left(\mathbb{R}^{3}\right)$. The fourth factor is a bounded operator in $H^{0}\left(\mathbb{R}^{3}\right)^{4}$ whose norm tends to zero as $z=i s$, $s \rightarrow \pm \infty$. The third factor is an operator bounded in the same space and independent of $z$. The second factor is a bounded operator in this space with norm tending to 0 . The first factor is an operator from this space to $H^{0}(\Gamma)^{4}$ not depending on $z$.

3.4. Now, we consider the operator

$$
|V|^{1 / 2}\left(L_{0}-z_{0}\right)^{-1}\left(L_{0}-z\right)^{-1}|V|^{1 / 2}
$$

(cf. (2.20)) under the assumption that $z$ and $z_{0}$ are not in the spectrum of $L_{0}$. This operator extends to a compact operator in $H^{0}(\Omega)^{4}$. To check this, it suffices to rewrite the above expression in the form

$$
\left\{|V|^{1 / 2}\left|L_{0}\right|^{-1 / 2}\right\}\left\{\left|L_{0}\right|^{1 / 2}\left(L_{0}-z_{0}\right)^{-1}\right\}\left\{\left|L_{0}\right|^{1 / 2}\left(L_{0}-z\right)^{-1}\right\}\left\{\left(|V|^{1 / 2}\left|L_{0}\right|^{-1 / 2}\right)^{*}\right\} .
$$

Here the second and third factors are compact. 
3.5. All this together allows us to use the results stated in Subsection 2.3.

By Proposition 2.6, the following is true.

Proposition 3.4. There exists a unique selfadjoint operator $\widetilde{L}$ in $H^{0}(\Omega)^{4}$ with $D(\widetilde{L}) \subset$ $D\left(\left|L_{0}\right|^{1 / 2}\right)=H_{1 / 2}$ such that

$$
\left(\widetilde{L} w_{1}, w_{2}\right)_{0, \Omega}=h_{L_{0}}\left[w_{1}, w_{2}\right]+h_{V}\left[w_{1}, w_{2}\right], \quad w_{1} \in D(\widetilde{L}), \quad w_{2} \in H_{1 / 2} .
$$

On the right-hand side of this formula it is possible to add the form (omitted previously) of the operator of multiplication by a smooth matrix $V_{1}$.

Theorem 3.5. The operators $L$ and $\widetilde{L}$ coincide.

Proof. We return to the definition of $L$. The domains of $L$ and $L_{0}$ are $H_{V}(\Omega, B)^{4}$ and $H^{1}(\Omega, B)^{4}$, respectively. Of course, the first domain contains the second one. Let $w_{1} \in H_{V}(\Omega, B)^{4}$. Then $\varphi_{1} w_{1}$ and $\varphi_{2} w_{1}$ are in $H_{V}(\Omega, B)^{4}$ as well (see, in particular, Proposition 2.3), and by (2.6) and (2.7) we have

$$
\begin{aligned}
L w_{1} & =(\mathcal{D}+(c+V))_{\mathbb{R}^{3}}\left(\varphi_{1} w_{1}\right)+\left(\mathcal{D}+c+\psi_{2} V\right)_{\Omega}\left(\varphi_{2} w_{1}\right) \\
& =\mathcal{D}\left(\varphi_{1} w_{1}\right)+(c+V)\left(\varphi_{1} w_{1}\right)+\mathcal{D}\left(\varphi_{2} w_{1}\right)+(c+V)\left(\varphi_{2} w_{1}\right) \\
& =L_{0} w_{1}+V w_{1} .
\end{aligned}
$$

Therefore,

$$
\left(L w_{1}, w_{2}\right)_{0, \Omega}=\left(\left(L_{0}+V\right) w_{1}, w_{2}\right)_{0, \Omega}=h_{L_{0}}\left[w_{1}, w_{2}\right]+h_{V}\left[w_{1}, w_{2}\right]
$$

for $w_{2} \in H_{1 / 2}$, and the operator $L$ coincides with $\widetilde{L}$ because the latter is unique.

Propositions 2.7 and 3.2 yield the following statement.

Proposition 3.6. 1. The function $z \mapsto(I+S M(z))^{-1}$ is a meromorphic operatorvalued function outside $\sigma\left(L_{0}\right)$. In particular, the operator $I+S M(z)$ has a bounded inverse everywhere outside $\sigma\left(L_{0}\right)$ except, possibly, a discrete set without finite limit points outside $\sigma\left(L_{0}\right)$.

2. Suppose $z$ does not belong to the spectrum $\sigma\left(L_{0}\right)$ and $I+S M(z)$ is an invertible operator. Then $z$ does not belong to $\sigma(L)$ and the formula

$$
\begin{aligned}
& (L-z)^{-1}-\left(L_{0}-z\right)^{-1} \\
& \quad=-\left(|V|^{1 / 2}\left(L_{0}-\bar{z}\right)^{-1}\right)^{*}(1+S M(z))^{-1} S|V|^{1 / 2}\left(L_{0}-z\right)^{-1}
\end{aligned}
$$

is true. In particular, there exists a real point $z=\lambda_{0}$ at which (3.27) is true.

Proof of Theorem 3.1. Consider the operator $K=|V|^{1 / 2}\left(L_{0}-\lambda_{0}\right)^{-1}$ with this $\lambda_{0}$. The spectrum of $K^{*} K$ coincides with the spectrum of the variational problem for the quotient of the quadratic forms $\|K w\|_{0, \Omega}^{2} /\|w\|_{0, \Omega}^{2}, w \in L_{2}(\Omega)^{4}$. Put $f=\left(L_{0}-\lambda_{0}\right)^{-1} w$; this is a function in $H^{1}(\Omega, B)^{4}$. We get a variational problem for the quotient

$$
(|V| f, f)_{0, \Omega} /\left\|\left(L_{0}-\lambda_{0}\right) f\right\|_{0, \Omega}^{2} \text {. }
$$

Here the denominator is equivalent to $\|f\|_{1, \Omega}^{2}$. The space $H^{1}(\Omega, B)^{4}$ lies between $H_{0}^{1}(\Omega)^{4}$ and $H^{1}(\Omega)^{4}$. If $T$ is a compact operator, we denote by $s_{j}(T)$ its $s$-numbers (singular numbers; see, e.g., [32] Chapter II]). It is easy to check that $|V| \in L_{3 / 2}(\Omega)$. By this, Theorem 3.1 in [6], and Theorem 1 in [10] on eigenvalues of variational problems, we have

so that

$$
s_{j}\left(K^{*} K\right)=O\left(j^{-2 / 3}\right)
$$

$$
s_{j}(K)=O\left(j^{-1 / 3}\right)
$$


Now we use the Ky Fan inequality for $s$-numbers of the product of compact operators $T_{1}$ and $T_{2}$,

$$
s_{k+l-1}\left(T_{1} T_{2}\right) \leq s_{k}\left(T_{1}\right) s_{l}\left(T_{2}\right),
$$

and the inequality $s_{j}(B T) \leq\|B\| s_{j}(T)$ for a compact operator $T$ and a bounded operator $B$ (see, e.g., 32]). From (3.27) we see that the difference of $\left(L-\lambda_{0}\right)^{-1}$ and $\left(L_{0}-\lambda_{0}\right)^{-1}$ is the product $K^{*} B K$, where $B$ is a bounded operator. Hence, the $s$-numbers of this difference can be estimated as $O\left(j^{-2 / 3}\right)$. As a consequence, the moduli of the eigenvalues of this difference are estimated in the same way. Since, by (9), the eigenvalues of $\left(L_{0}-\right.$ $\left.\lambda_{0}\right)^{-1}$ have asymptotics of the form $b^{-1} j^{-1 / 3}(j \rightarrow \pm \infty)$, the eigenvalues of $\left(L-\lambda_{0}\right)^{-1}$ have the same asymptotics by the version of the $\mathrm{H}$. Weyl theorem proposed by Birman and Solomyak (see Lemma 1.5 in [6]). This implies the statement of Theorem 3.1.

\section{$\S 4$. Calculation of the $R$-matrix: the first formula}

We describe a formal construction of the $R$-matrix by using the eigenfunctions of the first spectral problem. Consider the problem

$$
(\mathcal{D}+V) w=\lambda w \text { in } \Omega, \quad B w=g \text { on } \Gamma .
$$

Let $w$ be its solution for some $\lambda$ different from the eigenvalues $\lambda_{n}$. The function $w$ (as any function in $\left.L_{2}(\Omega)^{4}\right)$ can be expanded into a series with respect to the orthogonal basis $\left\{w_{n}\right\}_{1}^{\infty}$ of eigenfunctions:

$$
w=\sum_{-\infty}^{\infty} c_{n} w_{n} \quad \text { with } c_{n}=\left(w, w_{n}\right)_{\Omega} .
$$

Calculation of the coefficients via integration by parts shows that

$$
c_{n}=\frac{\left(g, u_{n}^{+}\right)_{\Gamma}}{\lambda_{n}-\lambda} .
$$

The details of this calculation are given at the end of this section. We obtain

$$
w(x)=\sum_{-\infty}^{\infty} \frac{\left(g, u_{n}^{+}\right)_{\Gamma}}{\lambda_{n}-\lambda} w_{n}(x)
$$

in $L_{2}(\Omega)^{4}$. If it were possible to take the values on the boundary in this expansion, we would get, in particular,

$$
u^{+}(x)=\sum_{-\infty}^{\infty} \frac{\left(g, u_{n}^{+}\right)_{\Gamma}}{\lambda_{n}-\lambda} u_{n}^{+}(x) .
$$

This is precisely the first formula for the $R$-matrix, i.e., for the operator transforming $g$ to $u^{+}$. As far as we know, the question of the validity of this expansion remained to be open for discussion in the physical literature (cf. 2] and the references therein). Unfortunately, we can also say nothing about this series even in the case of a smooth $g$. In order to pass to the boundary, we must view $w$ and $w_{n}$ as functions in $H^{t}(\Omega)^{4}$ with $t>1 / 2$, but then we arrive at a contradiction on the boundary, because $w_{n}$ satisfy the homogeneous boundary condition, while $w$ does not.

However, the following regularization of this series is possible. Mathematically, the trick is quite simple: we move the nonhomogeneity from the boundary condition into the equation. Let $g \in H^{1 / 2}(\Gamma)^{2}$, and let $w_{0}(x)$ be a function in $H^{1}(\Omega)^{4}$ with zero upper component $u_{0}$ and satisfying the boundary condition (7):

$$
i \sigma(\nu) v_{0}^{+}(x)=g(x) .
$$


If the potential has a singularity at the origin, then we assume additionally that $v_{0}(x)=0$ near the origin. The function $w-w_{0}$ belongs to the domain of the operator corresponding to the spectral problem. Its expansion in the eigenfunctions $w_{n}$ is convergent at least in $H^{1}\left(\Omega_{\varepsilon}\right)^{4}, \varepsilon>0$, and admits passage to the boundary. In particular, we get

$$
u^{+}(x)=\sum_{-\infty}^{\infty}\left[\frac{\left(g, u_{n}^{+}\right)_{\Gamma}}{\lambda_{n}-\lambda}-\left(v_{0}, v_{n}\right)_{\Omega}\right] u_{n}^{+}(x) .
$$

This expansion converges in $H^{1 / 2}(\Gamma)^{2}$. It should be noted that, actually, a series with zero sum in $\Omega$ on the level of $u$-components is subtracted from the series (4.4).

If no singularity is present, we can assume $g$ to be slightly more regular and obtain a better convergence: for $g \in H^{1-\delta}(\Gamma)^{2}$ we have the convergence in $H^{1-\delta}(\Gamma)^{2}$ with arbitrarily small $\delta>0$.

Now we present the omitted calculation. We have

$$
\begin{aligned}
\left((\mathcal{D}+V) w, w_{n}\right)_{\Omega}=\lambda\left(w, w_{n}\right)_{\Omega} & \\
& =i^{-1}\left[\left(\sigma(\nu) v^{+}, u_{n}^{+}\right)_{\Gamma}+\left(u^{+}, \sigma(\nu) v_{n}^{+}\right)_{\Gamma}\right]+\left(w,(\mathcal{D}+V) w_{n}\right)_{\Omega} \\
& =-\mu\left(u^{+}, u_{n}^{+}\right)_{\Gamma}-\left(g, u_{n}^{+}\right)_{\Gamma}+\mu\left(u^{+}, u_{n}^{+}\right)_{\Gamma}+\lambda_{n}\left(w, w_{n}\right)_{\Omega},
\end{aligned}
$$

hence $\left(\lambda-\lambda_{n}\right) c_{n}=-\left(g, u_{n}\right)_{\Gamma}$, and this yields (4.3).

\section{§5. Separation of variables in the Dirac equation WITH A SPHERICALLY SYMMETRIC POTENTIAL, AND the CASE OF THE COUlOMb SINGUlarity With $|\theta| \geq 1$}

In the case of a spherically symmetric potential and, in particular, for a pure Coulomb singularity, it is well known how to separate variables in the Dirac equation in $\mathbb{R}^{3}$ and to investigate the corresponding first order equation on a semiaxis. See the book 33 . by Weidmann and numerous references therein to earlier papers by Rellich, Weidmann, Roos-Sangren, and other authors. R. Szmytkowski called our attention to the fact that for the problem in the ball centered at the origin with the boundary condition (7) (where $g=0$ ), separation of variables works as well. Here we outline the changes that should be done in order to pass from $\mathbb{R}^{3}$ to a ball. Our notation differs a bit from that in [33].

5.1. Suppose the potential is a scalar function of the form $V=V(|x|)$. For simplicity, we consider the operator $\mathcal{D}+V$ in the unit ball $O_{1}=\{x:|x|<1\}$. We put

$$
\begin{aligned}
r & =|x|, \\
\sigma_{r} & =r^{-1} \sum x_{j} \sigma_{j}, \\
U & =U(x)=\left(\begin{array}{cc}
I_{2} & 0 \\
0 & -i \sigma_{r}
\end{array}\right) .
\end{aligned}
$$

The matrix $U(x)$ is unitary. By Theorem 1.A.2 in [33, we have

$$
U^{*} \mathcal{D} U=\left(\begin{array}{cc}
0 & -i I_{2} \\
i I_{2} & 0
\end{array}\right) P_{r}+\frac{i}{r}\left(\begin{array}{cc}
0 & i Q \\
i Q & 0
\end{array}\right)+\beta+V(|x|)
$$

where

$$
\begin{aligned}
P_{r} & =\frac{1}{r}\left(\sum_{1}^{3} x_{j} D_{j}-i\right), \\
Q & =I_{2}-i \sum_{1}^{3} \sigma_{j}(x \times \nabla)_{j} .
\end{aligned}
$$


In [33] the operator $Q$ was called the complete angular momentum. It can be regarded as an operator on the unit sphere $S^{2}$. This is a first order elliptic operator; its spectrum is discrete and is contained in $\mathbb{Z} \backslash\{0\}$ (compare with Theorem 1.A.3 in [33]). This is clear from the following formula checked in the proof:

$$
\left(Q-\frac{1}{2} I_{2}\right)^{2}=\frac{1}{4} I_{2}-\mathcal{B},
$$

where $\mathcal{B}$ is the Laplace-Beltrami operator on $S^{2}$ with the eigenvalues $l(l+1)$. Let $K_{l}$ be the corresponding $(4 l+2)$-dimensional eigenspace in $L_{2}\left(S^{2}\right)^{2}$ spanned by

$$
\left(\begin{array}{c}
Y_{l, j} \\
0
\end{array}\right),\left(\begin{array}{c}
0 \\
Y_{l, j}
\end{array}\right), \quad j=-l,-l+1, \ldots, l,
$$

where $Y_{l, j}$ is the complete orthonormal system of spherical harmonics of order $l$. This subspace is invariant with respect to $Q$, and the restriction $Q_{l}$ of this operator to $K_{l}$ has eigenvalues $l+1$ and $-l$ for $l \geq 1$. For $l=0$ the vectors in $K_{l}$ are constant, and we have only the eigenvalue 1 .

Thus, $Q$ has the following system of eigenfunctions orthonormal in $L_{2}\left(S^{2}\right)^{2}$ :

$$
Z_{k, j}=\left(\begin{array}{l}
z_{k, j, 1} \\
z_{k, j, 2}
\end{array}\right), \quad j=1, \ldots, j(k) ;
$$

they correspond to the eigenvalues $k \in \mathbb{Z} \backslash\{0\}$. Here $j(k)=2|k|$; see [34].

Denoting by $Y(r)$ the column of two scalar-valued functions $y_{1}(r)$ and $y_{2}(r)$, we define isometries

$$
W_{k, j}: L_{2}(0,1)^{2} \rightarrow L_{2}\left(O_{1}\right)^{4} \quad(k \in \mathbb{Z} \backslash\{0\}, j=1, \ldots, j(k))
$$

by the formula

$$
\left(W_{k, j} Y\right)(r \omega)=\left(\begin{array}{l}
r^{-1} y_{1}(r) Z_{k, j}(\omega) \\
r^{-1} y_{2}(r) Z_{k, j}(\omega)
\end{array}\right)=\left(\begin{array}{l}
r^{-1} y_{1}(r) z_{k, j, 1}(\omega) \\
r^{-1} y_{1}(r) z_{k, j, 2}(\omega) \\
r^{-1} y_{2}(r) z_{k, j, 1}(\omega) \\
r^{-1} y_{2}(r) z_{k, j, 2}(\omega)
\end{array}\right)
$$

Then

$$
L_{2}\left(O_{1}\right)^{4}=\bigoplus_{k, j} H_{k, j}, \quad \text { where } H_{k, j}=W_{k, j} L_{2}(0,1)^{2} .
$$

As a result (cf. Theorem 1.A.4 in 33]), we see that $\mathcal{D}+V$ is the orthogonal sum of the operators

$$
T_{k, j}=T_{k}=W_{k, j}^{*} U^{*} \mathcal{D} U W_{k, j}
$$

in $L_{2}(0,1)^{2}$ with

$$
T_{k} Y(r)=\left(\begin{array}{cc}
0 & -1 \\
1 & 0
\end{array}\right)\left(\begin{array}{l}
y_{1}^{\prime}(r) \\
y_{2}^{\prime}(r)
\end{array}\right)+\left(\begin{array}{cc}
V(r)+1 & -k r^{-1} \\
-k r^{-1} & V(r)-1
\end{array}\right)\left(\begin{array}{l}
y_{1}(r) \\
y_{2}(r)
\end{array}\right) .
$$

5.2. We return to our boundary-value problem and make the substitution

$$
w(x)=U\left(W_{k, j} Y\right)(r \omega)=r^{-1} U Y(r) Z_{k, j}(\omega)
$$

(see (5.7)). Obviously, $\sigma(x)=\sigma_{r}$ on the unit sphere. By the structure of our boundary condition (see $(7), g=0$ ) and of the matrix $U$, the variables separate, and we obtain the problem

$$
T_{k} Y(r)=\lambda Y(r) \quad(0<r<1), \quad y_{2}(1)=\mu y_{1}(1)
$$

on the interval $(0,1)$. In order to construct a selfadjoint extension of the operator corre- 
sponding to the initial problem in the ball, we need to construct a selfadjoint extension of the operator corresponding to the problem (5.12) for every $k$.

5.3. Now, let $V(r)=\theta / r$. The construction of a selfadjoint operator in $L_{2}(0,1)$ corresponding to the problem (5.12) is determined by the following facts. At the point $r=1$ we have the limit circle case. This means that for $0<c<1$ all solutions of the equation

$$
\left(T_{k}-\lambda\right) Y(r)=0
$$

on $(c, 1)$ are in $L_{2}(c, 1)^{2}$ for every $\lambda$. At the point 0 we have either the limit circle case or the limit point case. The latter means that for $0<c<1$ there exists a solution of $(5.13)$ on $(0, c)$ not belonging to $L_{2}(0, c)^{2}$. The domain of the corresponding selfadjoint operator consists of all (absolutely continuous) functions $Y(r)$ in $L_{2}(0,1)^{2}$ such that $T_{k} Y(r)$ belongs to $L_{2}(0,1)^{2}$. In the first case one boundary condition is needed at the point 0 and the other at the point 1 , while in the second case only one boundary condition at the point 1 is needed. The condition at the point 1 is already prescribed by separation of variables, and this is the boundary condition in (5.12).

Calculations show that at the point 0 the case of limit circle occurs if and only if

$$
k^{2}<\theta^{2}+\frac{1}{4}
$$

Since $k$ is a nonzero integer, for $|\theta| \leq \sqrt{3} / 2$ condition (5.14) fails for every $k$. Thus, it suffices to set the boundary condition at the point 1 . This illustrates the constructions of $\S 1$. If $\sqrt{3} / 2<|\theta|<1$, an additional condition is required at the point 0 only for $k= \pm 1$. This illustrates the constructions of $\S 2$. Here we see that the critical values $\sqrt{3} / 2$ and 1 for $|\theta|$ are quite essential.

As $|\theta|$ increases, more and more new values of $k$ appear requiring additional conditions at the point 0 . For the way of doing this, we refer the reader to the paper [34. We do not dwell on this, though the study of properties of every selfadjoint extension that can be constructed for a fixed $\theta$ with large $|\theta|$ is feasible.

\section{$\S 6$. Concluding Remarks}

The results of $\S \S 1-4$ can be generalized to the case of a potential with many poles. We only need to assume that the inequality for $\theta$ is fulfilled at every singular point, and to use a partition of unity consisting of several functions. Concerning the Dirac operator in $\mathbb{R}^{3}$ in this case; see, e.g., [35] and [36].

The operator $(\mathcal{D}+V)_{\Omega}$ introduced in $\S 2$ also admits another definition as a limit of operators with cut-off potentials $V^{(t)}$ instead of $V$. This idea can be realized by using the results of the papers [20]-[22] and 23, 24] for the Dirac operator with Coulomb potential in $\mathbb{R}^{3}$, at least in the case of a scalar potential. See the end of Subsection 2.1.

In the results of $\S \S 1-3$, the boundary condition (7) can be generalized. For example, $\mu$ can be replaced by a diagonal real matrix that has nonzero trace and depends smoothly on a point on $\Gamma$. It is not difficult to check that this is sufficient for the problem to be elliptic and formally selfadjoint.

Minimal smoothness assumptions to be imposed on the boundary and on the potential off the singularity in order to make our arguments working will not be analyzed here. Surely, the boundary need not be too smooth. But nonsmooth surfaces are not very interesting in the atomic physics, because there, in contrast to electrodynamics, there is a freedom in the choice of $\Gamma$. 


\section{ChAPTER II. THE PROBLEM WITH SPECTRAL PARAMETER IN THE BOUNDARY CONDITION}

\section{$\S 7$. Passage from the Dirac system to a SeCond order system, AND A HARDY-TYPE INEQUALITY}

In this chapter we shall not single out the parameter $\lambda$ in the Dirac system explicitly, and we write this system in the form

$$
\begin{aligned}
& (V(x)+1) u(x)+\sigma(D) v(x)=0, \\
& \sigma(D) u(x)+(V(x)-1) v(x)=0 .
\end{aligned}
$$

Here we shall first assume that $V(x)$ is a scalar function of the form

$$
V(x)=V_{0}(x)+V_{1}(x), \quad \text { where } V_{0}(x)=\frac{\theta}{|x|}, 0<|\theta|<1,
$$

and $V_{1}(x)$ is a smooth function; both are real-valued. In this chapter, for simplicity, when talking about the smoothness of $V_{1}(x)$ and of the boundary, we mean the infinite smoothness. Assume that the functions $V(x) \pm 1$ do not vanish in $\bar{\Omega}$ and hence have constant sign. For definiteness, let them be positive, so that $\theta>0$. Recall that the boundary condition in the spectral problem II is of the form (7) with $g=0$.

From the system (7.1) we get

$$
L_{1} u:=\sigma(D)(V-1)^{-1} \sigma(D) u-(V+1) u=0 .
$$

This is a homogeneous second order $(2 \times 2)$-system of Schrödinger type in a divergent form with degeneration in the leading term at one point 0 and a Coulomb singularity in the free term at the same point 5

Our analysis of the spectral problem II for the Dirac system with a singular potential will be based on the results for the Dirichlet problem

$$
L_{1} u=f \text { in } \Omega, \quad u^{+}=0 \text { on } \Gamma
$$

in a weak (variational) setting. They will be obtained in the next section. In [4 this problem was also used, but there all coefficients were smooth and system (7.3) had neither degeneration nor singularity.

Assuming the function $u$ to be smooth and vanishing at the boundary, we take the inner product of the left-hand side of (7.3) with $\bar{u}$ and integrate over $\Omega$. Integrating by parts and recalling that $\sigma(D)$ is a Hermitian operator, we obtain the quadratic form

$$
\Phi_{0}(u, u)=\int_{\Omega}(V-1)^{-1} \sigma(D) u \cdot \overline{\sigma(D) u} d x-\int_{\Omega}(V+1) u \cdot \bar{u} d x .
$$

Here we note the difference of signs: $(V-1)^{-1} \sim|x| / \theta$, but $-(V+1) \sim-\theta /|x|$ as $x \rightarrow 0$. We want to deduce a Gårding-type inequality for this form. To do this, we need the following Hardy-type inequality.

Proposition 7.1. For functions $u(x) \in C_{0}^{1}\left(\mathbb{R}^{3}\right)^{2}$, the following inequality is true:

$$
\int \frac{1}{|x|}|u|^{2} d x \leq \int|x||\sigma(\partial) u|^{2} d x
$$

Inequality (2.1) in 13 for a four-dimensional vector-valued function and the operator $\sum_{1}^{3} \alpha_{j} \partial_{j}$ immediately yields (7.6) if we let the two lower components of that vector-valued function be equal to zero.

\footnotetext{
${ }^{5}$ Any attempts to pass to a second order system in a different way did not lead to positive results.
} 
Corollary 7.2. For functions $u(x) \in C_{0}^{1}(\Omega)^{2}$, the following inequality is true:

$$
\|u\|_{L_{2}(\Omega)^{2}}^{2} \leq C \int_{\Omega}|x \| \sigma(\partial) u|^{2} d x .
$$

\section{$\S 8$. The Dirichlet Problem $(7.4)$}

We introduce the space $\widetilde{H}_{0}^{1}(\Omega)^{2}$ as the completion of the linear set $C_{0}^{\infty}(\Omega)^{2}$ with respect to the norm defined by

$$
\langle|u|\rangle_{1, \Omega}^{2}=\int_{\Omega}|x||\sigma(D) u|^{2} d x .
$$

(If $\langle|u|\rangle=0$, then $u=0$ by the uniqueness of a solution to the Dirichlet problem for the Laplace equation.) By (7.7), this space is continuously embedded into $L_{2}(\Omega)^{2}$.

Proposition 8.1. The space $\widetilde{H}_{0}^{1}(\Omega)^{2}$ is compactly embedded into $L_{2}(\Omega)^{2}$.

Proof. Fix an arbitrary $q \in(6 / 5,3 / 2)$. It suffices to check that $\widetilde{H}_{0}^{1}(\Omega)^{2}$ is continuously embedded into $W_{q}^{1}(\Omega)^{2}$. Indeed, the inequality $q>6 / 5$ implies the continuity of the embedding of $W_{q}^{1}(\Omega)$ into $H^{\varepsilon}(\Omega)$ with some positive $\varepsilon$ (see, e.g., [27], Section 4.6.1]), and the latter space is compactly embedded into $L_{2}(\Omega)$. Here the space $W_{q}^{1}(\Omega)$ is defined as the completion of the set of functions smooth in $\bar{\Omega}$ with respect to the norm defined by the formula (we write it for scalar-valued functions $h$ )

$$
\|h\|_{1, q, \Omega}^{q}=\sum\left\|\partial_{j} h\right\|_{L_{q}(\Omega)}^{q}+\|h\|_{L_{q}(\Omega)}^{q} .
$$

We need this norm in the current proof only.

Using the Hölder inequality, we get

$$
\begin{aligned}
& \int_{\Omega}|\sigma(\partial) u|^{q} d x=\int_{\Omega}\left[|x||\sigma(\partial) u|^{2}\right]^{q / 2}|x|^{-q / 2} d x \\
& \leq\left(\int_{\Omega}|x||\sigma(\partial) u|^{2} d x\right)^{q / 2}\left(\int_{\Omega}|x|^{-q /(2-q)} d x\right)^{(2-q) / 2} .
\end{aligned}
$$

The latter integral converges because $q<3 / 2$. Thus,

$$
\|\sigma(\partial) u\|_{L_{q}(\Omega)} \leq C_{1}\langle|u|\rangle_{1, \Omega} .
$$

Furthermore, by the Hölder inequality we have

$$
\|u\|_{L_{q}(\Omega)} \leq C_{2}\|u\|_{L_{2}(\Omega)} .
$$

It remains to use the a priori estimate by Agmon-Nirenberg for solutions to the elliptic system $\sigma(\partial) u=f$ in the linear set of smooth compactly supported functions on $\mathbb{R}^{3}$ with support lying inside a fixed compact set $(\bar{\Omega})$ :

$$
\|u\|_{1, q, \Omega} \leq C_{3}\left[\|\sigma(\partial) u\|_{L_{q}(\Omega)}+\|u\|_{L_{q}(\Omega)}\right] .
$$

Taking (7.7) into account, we obtain

$$
\|u\|_{1, q, \Omega} \leq C_{4}\langle|u|\rangle_{1, \Omega}
$$

as required.

Proposition 8.2. For functions $u \in \widetilde{H}_{0}^{1}(\Omega)^{2}$, the following Gairding-type inequality is true:

$$
\varepsilon\langle|u|\rangle_{1, \Omega}^{2} \leq\left[\Phi_{0}(u, u)+C\|u\|_{0, \Omega}^{2}\right]
$$

with some $\varepsilon>0$ and $C \geq 0$. 
Proof. Let $\varphi$ be a function in $C_{0}^{\infty}(\Omega)$ supported in $O_{\delta}$ with small $\delta$ and equal to 1 in a smaller neighborhood of the origin. We set $\psi=\left(1-\varphi^{2}\right)^{1 / 2}$. Let $u$ be an arbitrary function in $\widetilde{H}_{0}^{1}(\Omega)^{2}$. By Proposition 7.1, our assumptions on the potential imply that for $\delta$ sufficiently small the function $\varphi u$ satisfies (8.5) with some $\varepsilon_{1}$ and $C_{5}$ instead of $\varepsilon$ and $C$. Using (7.7) to estimate the terms with derivatives of $\varphi$, we obtain

$$
\varepsilon_{1}\langle|\varphi u|\rangle_{1, \Omega}^{2} \leq\left[\Phi_{0}(u, u)+C_{6}\|\varphi u\|_{0, \Omega}^{2}\right] .
$$

Furthermore, since the system $L_{1} u=f$ is strongly elliptic everywhere except the singularity, the function $\psi u$ satisfies the estimate

$$
\varepsilon_{2}\|\psi u\|_{1, \Omega}^{2} \leq\left[\Phi_{0}(\psi u, \psi u)+C_{7}\|\psi u\|_{0, \Omega}^{2}\right] .
$$

In its turn, this yields

$$
\varepsilon_{3}\langle|\psi u|\rangle_{1, \Omega}^{2} \leq\left[\Phi_{0}(u, u)+C_{8}\|u\|_{0, \Omega}^{2}\right] .
$$

The positive constants $\varepsilon_{j}$ and $C_{j}$ do not depend on $u$. It is clear that (8.6) and (8.7) imply the required result.

Now we consider the Dirichlet problem

$$
\left(L_{1}-\lambda\right) u=f \text { in } \Omega, \quad u^{+}=0,
$$

in the weak setting

$$
\Phi_{\lambda}(u, v)=: \Phi_{0}(u, v)-\lambda(u, v)_{0, \Omega}=(f, v)_{0, \Omega} \quad \text { for } v \in \widetilde{H}_{0}^{1}(\Omega)^{2},
$$

where the form $\Phi_{0}(u, v)$ is defined in an obvious way. It is standard to prove that, by (8.5), this problem has a unique solution in $\widetilde{H}_{0}^{1}(\Omega)^{2}$ for sufficiently large absolute values of $\lambda$ and for arbitrary $f$, in particular, for $f$ in $H^{0}(\Omega)^{2}$. Namely, if we take $\Phi_{\lambda}(u, v)$ as the inner product in $\widetilde{H}_{0}^{1}(\Omega)^{2}$, then the continuous antilinear functional $v \mapsto(f, v)$ on this space has a unique representation in the form $\Phi_{\lambda}(u, v)$.

The a priori estimate can be written at least in the form

$$
\langle|u|\rangle_{1, \Omega} \leq C_{9}\|f\|_{0, \Omega} \text {. }
$$

Remark 8.3. A similar result can be obtained, for example, for the scalar equation

$$
\nabla(|x| \nabla u)+\theta^{2}|x|^{-1} u=0, \quad 0<\theta<1,
$$

and for systems having a similar (and more general) structure with degeneration in the principal term and singularity in the lower order term. The Dirichlet problem for elliptic systems with degeneration on the boundary was treated in many papers; however, some other types of degeneration were considered as well. Cf. the papers [6] and [10] we have already mentioned in connection with spectral asymptotics.

Now, as in [1, Chapter VI, §2], with the problem (7.1) we can associate a selfadjoint operator $\mathcal{L}_{1}$ in $L_{2}(\Omega)^{2}$ semibounded below, with domain $D\left(\mathcal{L}_{1}\right)$ contained in $\widetilde{H}_{0}^{1}(\Omega)^{2}$ and such that

$$
\left(\mathcal{L}_{1} u, v\right)_{0, \Omega}=\Phi_{0}(u, v) \text { for } u \in D\left(\mathcal{L}_{1}\right), v \in \widetilde{H}_{0}^{1}(\Omega)^{2} .
$$

This operator is uniquely determined by the conditions listed above. By Proposition 8.1 the spectrum of this operator is discrete, and the following assumption (to be added from now on) is reasonable: the Dirichlet problem for the system $L_{1} u=f$ in the weak sense cannot have two different solutions.

Unique solvability is carried over to the case of a nonhomogeneous boundary condition $\left.u\right|_{\Gamma}=u^{+} \in H^{1 / 2}(\Gamma)^{2}$ in a usual way, namely, by the consideration of the difference between the solution and a function $u_{0}$ belonging to $H^{1}(\Omega)^{2}$, having this boundary value, and vanishing near the singularity. The solution of the homogeneous equation 
$L_{1} u=0$ in $\Omega_{0}$ with the nonhomogeneous boundary condition $\left.u\right|_{\Gamma}=u^{+}$belongs to the space $\widetilde{H}^{1}(\Omega)^{2}$. This is the completion of the linear set $C^{\infty}(\bar{\Omega})^{2}$ with respect to the norm defined by the formula

$$
\langle\langle|u|\rangle\rangle_{1, \Omega}^{2}=\langle|u|\rangle_{1, \Omega}^{2}+\|u\|_{0, \Omega}^{2} .
$$

We obtain the a priori estimate

$$
\langle\langle|u|\rangle\rangle_{1, \Omega} \leq C_{10}\left\|u^{+}\right\|_{1 / 2, \Gamma} .
$$

Indeed, we may assume that $\left\|u_{0}\right\|_{1, \Omega} \leq C_{11}\left\|u^{+}\right\|_{1 / 2, \Gamma}$; then the function $L_{1} u_{0}=f$ belongs to $H^{-1}(\Omega)^{2}$, and moreover, it vanishes near the singularity. It gives rise to a continuous antilinear functional on $\widetilde{H}_{0}^{1}(\Omega)^{2}$; thus, the equation $L_{1} u_{1}=-f$ with the homogeneous Dirichlet condition $u_{1}^{+}=0$ has a solution in $\widetilde{H}_{0}^{1}(\Omega)^{2}$, and for it we have

$$
\left\langle\left|u_{1}\right|\right\rangle_{1, \Omega} \leq C_{12}\|f\|_{-1, \Omega} \leq C_{13}\left\|u_{0}\right\|_{1, \Omega} .
$$

We set $u=u_{0}+u_{1}$.

Remark 8.4. If $\mathcal{L}_{1}-\lambda I$ is a nonnegative operator (this is true for negative $\lambda<0$ with sufficiently large absolute value), then the domain of its nonnegative square root is the space $\widetilde{H}_{0}^{1}(\Omega)^{2}$. See again [1] Chapter VI, §2].

The following proposition can easily be checked by applying the theorem on local smoothness for solutions of elliptic problems.

Proposition 8.5. For arbitrarily small $\varepsilon>0$ and $s \geq 1 / 2$, if $u^{+} \in H^{s}(\Gamma)^{2}$, then the solution of the system $L_{1} u=0$ belongs to $H^{s+1 / 2}\left(\Omega_{\varepsilon}\right)^{2}$. In particular, this solution belongs to $C^{\infty}(\bar{\Omega} \backslash\{0\})^{2}$ if $u^{+} \in C^{\infty}(\Gamma)^{2}$.

Furthermore, the Poisson operator $\mathcal{P}_{1}$ is well defined; it maps $u^{+}$to the solution of the homogeneous system $L_{1} u=0$ in $\Omega_{0}$.

Proposition 8.6. The Poisson operator $\mathcal{P}_{1}$ maps $H^{s}(\Gamma)^{2}$ to $H^{s+1 / 2}\left(\Omega_{\varepsilon}\right)^{2}$ boundedly for $s \geq 1 / 2$ and arbitrarily small $\varepsilon>0$ :

$$
\|u\|_{s+1 / 2, \Omega_{\varepsilon}} \leq C_{14}(\varepsilon)\left\|u^{+}\right\|_{s, \Gamma} .
$$

In this proposition nothing is said about the smoothness of the solution on the inner part of the boundary of the domain $\Omega_{\varepsilon}$, i.e., on the sphere of radius $\varepsilon$ centered at the origin. But in fact, when assuming that $u^{+} \in H^{s}(\Gamma)^{2}$, we automatically have the same smoothness on this sphere. Here our results for the Dirichlet problem (7.4) are essential.

Proof of Proposition 8.6. Let $\psi$ be a function in $C^{\infty}(\bar{\Omega})$ vanishing in $O_{\varepsilon / 2}$ and equal to 1 outside $O_{\varepsilon}$. We can write a priori estimates for Sobolev norms of $\psi u$ in terms of Sobolev norms of the boundary function. Let $u^{+} \in H^{s}(\Gamma)^{2}$; then $\psi u \in H^{s+1 / 2}\left(\Omega_{\varepsilon / 4}\right)^{2}$. We obtain

$$
\|\psi u\|_{s+1 / 2, \Omega_{\varepsilon / 4}} \leq C_{15}\left[\left\|L_{1}(\psi u)\right\|_{s-3 / 2, \Omega_{\varepsilon}}+\left\|u^{+}\right\|_{s, \Gamma}\right]
$$

consequently,

$$
\|u\|_{s+1 / 2, \Omega_{\varepsilon}} \leq C_{16}\left[\|u\|_{s-1 / 2, \Omega_{\varepsilon}}+\left\|u^{+}\right\|_{s, \Gamma}\right]
$$

whence

$$
\|u\|_{s+1 / 2, \Omega_{\varepsilon}} \leq C_{17}\left[\|u\|_{0, \Omega}+\left\|u^{+}\right\|_{s, \Gamma}\right] .
$$

But, by (8.13) and (8.12), the zero order norm of the solution is estimated by $\left\|u^{+}\right\|_{1 / 2, \Gamma}$. As a result, we arrive at (8.14). 


\section{§9. The OPERATOR ON $\Gamma$ CORRESPONDING TO THE SPECTRAL PROBLEM}

Now it is natural to consider system (7.1) under the following assumptions: $u, v \in$ $\widetilde{H}^{1}(\Omega)^{2}$, i.e.,

$$
|x|^{1 / 2} \sigma(\partial) u,|x|^{1 / 2} \sigma(\partial) v \in L_{2}(\Omega)^{2} .
$$

By (7.6) this yields

$$
|x|^{-1 / 2} u,|x|^{-1 / 2} v \in L_{2}(\Omega)^{2} .
$$

This agrees with system (7.1) if we rewrite it in the form

$$
\begin{aligned}
& (V+1)^{1 / 2} u+(V+1)^{-1 / 2} \sigma(D) v=0, \\
& (V-1)^{-1 / 2} \sigma(D) u+(V-1)^{1 / 2} v=0,
\end{aligned}
$$

in the sense that all terms in these equations belong to $L_{2}(\Omega)^{2}$. Here, moreover, $u, v \in$ $C^{\infty}(\bar{\Omega} \backslash\{0\})^{2}$.

System (7.1) is formally equivalent to the system consisting of (7.3) and the second equation in (7.1). Indeed, they imply the first equation in (7.1).

Since $V$ is assumed to be scalar, from the second equation in (7.1) we see that

$$
i \sigma(\nu) v^{+}=\mathcal{Q} u^{+}, \quad \text { where } \mathcal{Q}:=-\left[(V-1)^{-1} \sigma(\nu) \sigma(\partial) \mathcal{P}_{1} \cdot\right]^{+} .
$$

For this operator we have the following result. In its statement the same coordinate system is used as in [4], i.e., the origin is placed at a point on $\Gamma$ and the axis $x_{3}$ is directed along the outward normal at this point. At the end of the section, we explain why such a choice of the coordinate system does not restrict generality.

Proposition 9.1. The operator $\mathcal{Q}$ is a first order pseudodifferential operator with principal symbol given by

$$
a_{1}\left(\xi^{\prime}\right)=-(V-1)^{-1}\left[\left|\xi^{\prime}\right|+i \sum_{k \neq 3} \xi_{k} \sigma_{3} \sigma_{k}\right] .
$$

Proof. First, we explain why $\mathcal{Q}$ is a pseudodifferential operator. Let $\widetilde{L}_{1}$ be a strongly elliptic second order matrix operator with coefficients infinitely smooth in $\bar{\Omega}$ and coinciding with the coefficients of $L_{1}$ in a strip near the boundary. We compare the corresponding Poisson operators $\mathcal{P}_{1}$ and $\widetilde{\mathcal{P}}_{1}$. It suffices to show that their difference is an infinitely smoothing operator in the boundary strip. We consider the difference $u=\mathcal{P}_{1} g-\widetilde{\mathcal{P}}_{1} g$, where, say, $g \in H^{1 / 2}(\Gamma)^{2}$, and apply the operator $\widetilde{L}_{1}$ to this difference. In our boundary strip we have $\widetilde{L}_{1} u=L_{1} \mathcal{P}_{1} g=0$. Moreover, $u^{+}=0$. By the theorem on local smoothness for solutions of elliptic boundary-value problems, the function $u$ is infinitely smooth in the boundary strip.

Now, calculating the principal symbol of the operator $\mathcal{Q}$, we freeze the coefficients and assume that we deal with the operator $\beta \sigma(\partial) \sigma(\partial)=\beta \Delta I_{2}$ instead of $L_{1}$. Here the nonzero constant $\beta$ plays no role, because the Poisson operator transforms the Dirichlet data to a solution of the homogeneous equation; so, let $\beta=1$. This is precisely the case considered in [4, §4]. In the notation of that paper, we need the operator

$$
-(V-1)^{-1}\left[-\frac{1}{2} A^{-1}+c(\partial)\right]
$$

up to terms of zero order. The principal symbol of the operator $-\frac{1}{2} A^{-1}$ is $\left|\xi^{\prime}\right| I_{2}$, and that of the operator $c(\partial)$ is $i \sum_{k \neq 3} \sigma_{3} \sigma_{k} \xi_{k}$. 
In what follows we write $Q=Q_{1}$ instead of $Q$.

Now we observe that, instead of system (7.3), we could start with a similar second order system for $v$ :

$$
L_{2} v:=\sigma(D)(V+1)^{-1} \sigma(D) v-(V-1) v=0 .
$$

Assume that the Dirichlet problem in $\Omega$ for this system also cannot have two different solutions in the weak sense. Denote the corresponding Poisson operator by $\mathcal{P}_{2}$. Using this operator as above, we can first construct an operator expressing $i \sigma(\nu) u^{+}$in terms of $v^{+}$:

It follows that

$$
i \sigma(\nu) u^{+}=-\left[(V+1)^{-1} \sigma(\nu) \sigma(\partial) \mathcal{P}_{2} v^{+}\right]^{+} .
$$

$$
u^{+}=i \mathcal{Q}_{2} \sigma(\nu) v^{+}, \quad \text { where } \mathcal{Q}_{2}=\sigma(\nu)\left[(V+1)^{-1} \sigma(\nu) \sigma(\partial) \mathcal{P}_{2} \sigma(\nu)\right]^{+} .
$$

For this operator we obtain an analog of Proposition 9.1 with the only difference that, instead of (9.5), we get the following formula for the principal symbol:

$$
a_{2}\left(\xi^{\prime}\right)=(V+1)^{-1}\left[\left|\xi^{\prime}\right|+i \sum_{k \neq 3} \xi_{k} \sigma_{k} \sigma_{3}\right] .
$$

As in 4], the operators $\mathcal{Q}_{1}$ and $\mathcal{Q}_{2}$ are mutually inverse on the set of infinitely smooth functions. They are not elliptic.

We give yet another explanation of this fact. If we eliminate $v^{+}$from the boundary condition (7), we obtain the problem

$$
L_{1} u=0 \text { in } \Omega, \quad-\sigma(\nu)(V-1)^{-1} \sigma(\nu) u^{+}-\mu u^{+}=g .
$$

We may assume that it was reduced to the equation in (9.4). This problem resembles the classical Steklov problem

$$
\Delta u=0 \text { in } \Omega, \quad \partial_{\nu} u^{+}-\mu u^{+}=g
$$

but, in contrast to the latter, is readily seen to be nonelliptic.

Recall that we assume $V \pm 1$ to be positive functions.

Theorem 9.2. For arbitrary nonzero real $\alpha$ and $\beta$, the pseudodifferential operator $\mathcal{S}_{\alpha \beta}=$ $\alpha \mathcal{Q}_{1}+\beta \mathcal{Q}_{2}$ is elliptic. Its principal symbol has only positive eigenvalues for $\beta>0>\alpha$ and eigenvalues of both signs for $\alpha \beta>0$.

Proof. Consider a fixed boundary point and a coordinate system with origin at this point, the same as above. At this boundary point, we set

$$
\begin{aligned}
& (V-1)^{-1} \alpha=\alpha_{1}, \\
& (V+1)^{-1} \beta=\beta_{1} .
\end{aligned}
$$

It is easy to check that the principal symbol of the operator $\mathcal{S}_{\alpha \beta}$ is the Hermitian matrix

$$
\left(\begin{array}{cc}
\left(-\alpha_{1}+\beta_{1}\right)\left|\xi^{\prime}\right| & \left(-\alpha_{1}-\beta_{1}\right)\left(i \xi_{1}+\xi_{2}\right) \\
\left(-\alpha_{1}-\beta_{1}\right)\left(-i \xi_{1}+\xi_{2}\right) & \left(-\alpha_{1}+\beta_{1}\right)\left|\xi^{\prime}\right|
\end{array}\right) .
$$

Its determinant is $-4 \alpha_{1} \beta_{1}\left|\xi^{\prime}\right|^{2}$, thus the operator $\mathcal{S}_{\alpha \beta}$ is elliptic. Its eigenvalues are $2 \beta_{1}\left|\xi^{\prime}\right|$ and $-2 \alpha_{1}\left|\xi^{\prime}\right|$. This implies the remaining assertions of the theorem.

Now we return to our choice of the coordinate system. It is easy to check that, under a rotation of the coordinate system in $\mathbb{R}^{3}$, the three matrices $\sigma_{1}, \sigma_{2}, \sigma_{3}$ are transformed to matrices that remain to be Hermitian, satisfy relations (4), and have the eigenvalues \pm 1 . It is known that, by a unitary transformation of the variables $u_{1}, u_{2}$ (and by multiplication of the system from the left by the matrix of the inverse transformation), we can return these three matrices to their initial form $\sigma_{1}, \sigma_{2}, \sigma_{3}$ (see, e.g., [37] Part II, 
Appendix C]; compare with [38, Part I, §9]). Precisely this argument shows that we do not restrict generality by using the above coordinate system.

\section{$\S 10$. Spectral properties of the operator $\mathcal{Q}$}

The following considerations refine those in [4. This refinement is based on Theorem 9.2 absent in [4]. Only the operator $\mathcal{S}$ alone was considered in that paper, which works, for instance, in the case of a free Dirac operator.

The operator $\mathcal{Q}$ is formally selfadjoint in $L_{2}(\Gamma)^{2}$. As in [4, this can be verified by integration by parts in $\Omega$. Therefore, every operator $\mathcal{S}_{\alpha \beta}$ is formally selfadjoint as well. But it is a first order elliptic pseudodifferential operator, and hence $\mathcal{S}_{\alpha \beta}$ can be viewed as a selfadjoint operator in $L_{2}(\Gamma)^{2}$ with domain $H^{1}(\Omega)^{2}$. It has an orthonormal basis of eigenfunctions in $L_{2}(\Gamma)^{2}$, which remains to be an unconditional basis in all Sobolev spaces $H^{s}(\Gamma)^{2}$.

We take 1) $\alpha=\beta=1$ and 2) $\alpha=-1, \beta=1$, i.e., we consider the operators $\mathcal{Q}^{-1}+\mathcal{Q}$ and $\mathcal{Q}^{-1}-\mathcal{Q}$. We redenote them by $\mathcal{S}_{1}$ and $\mathcal{S}_{2}$.

The operator $\mathcal{S}_{1}$ has eigenvalues of both signs tending to $\pm \infty$. Numbered monotonically with their multiplicities taken into account, the eigenvalues have the asymptotics

$$
\tau_{n}^{(1)}= \pm c_{1}^{ \pm}|n|^{1 / 2}+O(1)(n \rightarrow \pm \infty) .
$$

The eigenvalues of $\mathcal{S}_{2}$ tend to $+\infty$. Numbered in nondecreasing order, they have the asymptotics

$$
\tau_{n}^{(2)}=c_{2} n^{1 / 2}+O(1) \quad(n \rightarrow+\infty) .
$$

Here $c_{1}^{ \pm}$and $c_{2}$ are positive constants. See [39], where a method of calculation of these constants can be found. We shall not dwell on this.

For every eigenvalue $\tau$ the corresponding eigenspace of $\mathcal{S}_{1}$ is finite-dimensional, consists of infinitely smooth functions, and is invariant with respect to the operator $\mathcal{Q}$ that commutes with $\mathcal{S}_{1}$ and satisfies the relation $\mathcal{Q}^{-1}+\mathcal{Q}=\mathcal{S}_{1}$. Therefore, $\mathcal{Q}$ has an orthonormal basis of eigenfunctions there, and the eigenvalues are the roots of the quadratic equation

$$
\mu^{-1}+\mu=\tau
$$

We have a similar situation for the eigenspaces of $\mathcal{S}_{2}$, and the corresponding quadratic equation is

$$
\mu^{-1}-\mu=\tau
$$

Consider the root sequence $\tau_{n}^{(1)}$ in (10.1) with $n>0$, which tends to $+\infty$. The formula for the roots of equation (10.3), namely,

$$
\mu=\frac{1}{2}\left(\tau \pm \sqrt{\tau^{2}-4}\right)
$$

implies that at least one of the points $+\infty$ or +0 is a limit point of the corresponding eigenvalues $\mu$ for the operator $Q$, but at this moment it is still unclear whether both of them are limit points. Here +0 means that the eigenvalues tend to 0 from the right. No more limit points may appear. If we assume that some subsequence of the eigenvalues (10.5) tends to $+\infty$, then (10.4) implies that the corresponding eigenvalues $\tau$ of $\mathcal{S}_{2}$ tend to $-\infty$, which is impossible. Therefore, for all positive $n$ except, perhaps, a finite number of them, the minus sign should be taken in (10.5) and the corresponding eigenvalues of $\mathcal{Q}$ tend to +0 with recalculation of the asymptotic behavior by (10.1) and (10.5).

Now we consider the sequence of eigenvalues $\tau_{n}^{(1)}$ of $\mathcal{S}_{1}$ with negative $n$. It tends to $-\infty$. From (10.5) we see that the corresponding eigenvalues of $\mathcal{Q}$ might have $-\infty$ and/or -0 as limit points. The second possibility contradicts the fact that the right-hand side 
of (10.4) may tend to $+\infty$ only. Therefore, for all negative $n$ except, probably, a finite number of them, the eigenvalues (10.5) of $\mathcal{Q}$ correspond to the "plus" sign in (10.5) (again with recalculation of the asymptotic behavior by using (10.1) and (10.5)).

Thus, the eigenvalues of $\mathcal{Q}$ have two limit points, +0 and $-\infty$. The coefficients of the principal terms in the asymptotics can easily be expressed in terms of $c_{1}^{ \pm}$(see the theorem below). The remainder estimate follows from the remainder estimate in (10.1) and from the asymptotics for the roots of equation (10.5). We arrive at the following main result.

Theorem 10.1. The eigenfunctions of the operator $\mathcal{Q}$ are infinitely smooth and form an orthonormal basis in $L_{2}(\Gamma)^{2}$ that remains to be an unconditional basis in all Sobolev spaces $H^{s}(\Gamma)^{2}$. The corresponding eigenvalues split in two sequences, one of them tends to +0 and the other to $-\infty$.

More precisely, these series of eigenvalues $\left\{\mu_{n}^{ \pm}\right\}$have the following asymptotic behavior:

$$
\begin{aligned}
& \mu_{n}^{+}=\left(c_{1}^{+}\right)^{-1} n^{-1 / 2}+O\left(n^{-1}\right)(n \rightarrow \infty), \\
& \mu_{n}^{-}=-c_{1}^{-}|n|^{1 / 2}+O(1)(n \rightarrow-\infty) .
\end{aligned}
$$

Thus, the operator $\mathcal{Q}$ is the orthogonal sum of an operator with discrete spectrum (i.e., with compact resolvent) in a subspace of $L_{2}(\Gamma)^{2}$ and a compact operator in another subspace. The sum of these two mutually orthogonal subspaces is $L_{2}(\Gamma)^{2}$. There is no uniqueness: any finite set of eigenspaces can be attributed arbitrarily to any of these two subspaces.

If $u^{+}$is an eigenfunction of $\mathcal{Q}$, then $v^{+}$can be reconstructed by the formula $v^{+}=$ $-i \sigma(\nu) \mathcal{Q} u^{+}$. After this, we reconstruct $u$ and $v$ in $\Omega$ by the formulas $u=\mathcal{P}_{1} u^{+}$and $v=\mathcal{P}_{2} v^{+}$. These functions possess properties (9.1) and belong to $C^{\infty}(\bar{\Omega} \backslash\{0\})^{2}$.

\section{$\S 11$. Second formula for the $R$-matrix}

This formula is simply the construction of the required operator $g \mapsto u^{+}$by expansion of $g$ and $u^{+}$in eigenfunctions of the operator $\mathcal{Q}$. If $\left\{\varphi_{n}\right\}_{-\infty}^{\infty}$ is the orthonormal basis in $L_{2}(\Gamma)^{2}$ consisting of these eigenfunctions, then

$$
g=\sum_{-\infty}^{\infty}\left(g, \varphi_{n}\right)_{\Gamma} \varphi_{n}
$$

and for $\mu$ different from the eigenvalues $\mu_{n}$ we obtain

$$
u^{+}=\sum_{-\infty}^{\infty} \frac{\left(g, \varphi_{n}\right)_{\Gamma}}{\mu_{n}-\mu} \varphi_{n}
$$

Here convergence improves together with the smoothness of $u^{+}$, and the latter increases together with that of $g$. Even a moderate smoothness of $g$ guarantees the uniform convergence: it suffices to assume $g \in H^{1+\varepsilon}(\Gamma)^{2}, \varepsilon>0$, because the convergence in $H^{1+\varepsilon}(\Gamma)^{2}$ on a two-dimensional surface implies the uniform convergence.

By (8.13), the approximations of $u^{+}$correspond to approximations of the solution $u$ in $\Omega_{\varepsilon}$ (with an arbitrarily small $\varepsilon$ ) with respect to the Sobolev norms of arbitrarily high order.

\section{§12. ConCluding Remarks}

The results of this chapter can also be generalized to the case of a potential with many poles. Some matrix-valued $V$ are acceptable as well. We shall not dwell on this here. 
In the case of the problem in the ball with purely Coulomb potential, the eigenfunctions can be written explicitly (cf. §5). They are expressed via spherical harmonics.

Without the assumption about unique solvability of the Dirichlet problem for the second order systems, these problems are still Fredholm, and a possibility remains to construct a basis of eigenfunctions with finite-dimensional defect.

Surely, in the considerations of this chapter, the assumptions about the smoothness of the boundary and of the potential outside the singularity can be relaxed.

\section{REFERENCES}

[1] T. Kato, Perturbation theory for linear operators, Grundlehren Math. Wiss., vol. 132, SpringerVerlag, Berlin-New York, 1976. MR0407617(53:11389)

[2] R. Szmytkowski, Metoda R-macierzy dla róvnań Schrödingera i Diraca, Politechnika Gdańska, Gdańsk, 1999.

[3] R. Courant and D. Hilbert, Methods of mathematical physics. Vol. 1, Intersci. Publishers, Inc., New York, NY, 1953. MR0065391 (16:426a)

[4] M. S. Agranovich, Spectral problems for the Dirac system with a spectral parameter in the local boundary conditions, Funktsional. Anal. i Prilozhen. 35 (2001), no. 3, 1-18; English transl., Funct. Anal. Appl. 35 (2001), no. 3, 161-175. MR1864984 (2003a:81034)

[5] V. Ivrii, Precise spectral asymptotics for elliptic operators acting in fiberings over manifolds with boundary, Lecture Notes in Math., vol. 1100, Springer-Verlag, Berlin, 1984. MR0771297 (86h:58139)

[6] M. Sh. Birman and M. Z. Solomyak, Spectral asymptotics of nonsmooth elliptic operators. I, II, Trudy Moskov. Mat. Obshch. 27 (1972), 3-52; ibid. 28 (1973), 3-34; English transl., Trans. Moscow Math. Soc. 27 (1975), 1-52; ibid. 28 (1975), 1-32. MF 0364898 (51:1152)

[7] B. Thaller, The Dirac equation, Springer-Verlag, Berlin, 1992. MR1219537(94k:81056)

[8] H. Kalf, U.-W. Schmincke, J. Walter, and R. Wüst, On the spectral theory of Schrödinger and Dirac operators with strongly singular potentials, Spectral Theory and Differential Equations (Proc. Sympos., Dundee, 1974), Lecture Notes in Math., vol. 448, Springer-Verlag, Berlin, 1975, pp. 182226. MR0397192 (53:1051)

[9] G. Nenciu, Self-adjointness and invariance of the essential spectrum for Dirac operators defined as quadratic forms, Comm. Math. Phys. 48 (1976), 235-247. MR0421456 (54:9459)

[10] G. Rozenblum, Distribution of the discrete spectrum of singular differential operators, Izv. Vyssh. Uchebn. Zaved. Mat. 1976, no. 1 (164), 75-80; English transl., Soviet Math. (Izv. VUZ) 20 (1976), no. 1, 63-71. MR0430557(55:3562)

[11] G. Nakamura and T. Tsuchida, Uniqueness for an inverse boundary value problem for Dirac operators, Comm. Partial Differential Equations 25 (2000), no. 7-8, 1327-1369. MR1765140 (2001g:35265)

[12] J. Dolbeault, M. J. Esteban, and E. Séré, On the eigenvalues of operators with gaps. Application to Dirac operators, J. Funct. Anal. 174 (2000), 208-226. MR1761368|(2001e:47040)

[13] V. Vogelsang, Remark on essential selfadjointness of Dirac operators with Coulomb potentials, Math. Z. 196 (1987), 517-521. MR0917234 (88k:35149)

[14] F. J. Narcowich, Mathematical theory of R-matrix. I. The eigenvalue problem; II. The R-matrix and its properties, J. Math. Phys. 15 (1974), no. 10, 1626-1634; 1635-1642. MR0386536 (52:7390a)

[15] M. S. Agranovich, Spectral problems for second-order strongly elliptic systems in domains with smooth and nonsmooth boundaries, Uspekhi Mat. Nauk 57 (2002), no. 5, 3-78; English transl., Russian Math. Surveys 57 (2002), no. 5, 847-920. MR1992082 (2004e:35168)

[16] P. Hamacher and J. Hinze, Finite-volume variational method for the Dirac equation, Phys. Rev. A (3) 44 (1991), no. 9, 1705-1711. MR1121458 (92d:81039)

[17] U.-W. Schmincke, Essential selfadjointness of Dirac operators with a strongly singular potential, Math. Z. 126 (1972), 71-81. MR0304896 (46:4028)

[18] A. M. Boutet de Monvel and R. Purice, A distinguished self-adjoint extension for the Dirac operator with strong local singularities and arbitrary behaviour at infinity, Rep. Math. Phys. 34 (1994), 351360. MR0304896 (46:4028)

[19] M. S. Agranovich, Elliptic boundary problems, Partial Differential Equations, 9, Encyclopaedia Math. Sci., vol. 79, Springer-Verlag, Berlin, 1997, pp. 1-144. MR1481215 (99a:35056)

[20] R. Wüst, A convergence theorem for selfadjoint operators applicable to Dirac operators with cutoff potentials, Math. Z. 131 (1973), 339-349. MR0355655 (50:8129)

[21] _ Distinguished self-adjoint extensions of Dirac operators constructed by means of cut-off potentials, Math. Z. 141 (1975), 93-98. MR0365233 (51:1486) 
[22] _ Dirac operations with strongly singular potentials, Math. Z. 152 (1977), 259-271. MR.0437948 (55:10869)

[23] M. Klaus and R. Wüst, Characterization and uniqueness of distinguished selfadjoint extensions of Dirac operators, Comm. Math. Phys. 64 (1979), 171-176. MR.0519923 (80k:81025)

[24] Spectral properties of Dirac operators with singular potentials, J. Math. Anal. Appl. 72 (1979), 206-214. MR0552332 (81b:35082)

[25] U.-W. Schmincke, Distinguished selfadjoint extensions of Dirac operators, Math. Z. 129 (1972), 335-349. MR0326448 (48:4792)

[26] M. A. Krasnosel'skiı̆, P. P. Zabrě̌ko, E. I. Pustyl'nik, and P. E. Sobolevskiǐ, Integral operators in spaces of summable functions, "Nauka", Moscow, 1966; English transl., Noordhoff Internat. Publishing, Leiden, 1976. MR0206751 (34:6568)

[27] H. Triebel, Interpolation theory, function spaces, differential operators, VEB Deutscher Verlag Wiss., Berlin, 1978. MR0500580 (80i:46032a)

[28] P. Grisvard, Caractérisation de quelques espaces d'interpolation, Arch. Rational Mech. Anal. 25 (1967), 40-63. MR 0213864 (35:4718)

[29] R. T. Seeley, Interpolation in $L_{p}$ with boundary conditions, Studia Math. 44 (1972), 47-60. MR0315432 (47:3981)

[30] L. R. Volevich and S. G. Gindikin, Generalized functions and convolution equations, "Nauka", Moscow, 1994. (Russian) MR1379334 (97g:46041)

[31] M. Sh. Birman, Scattering problems for differential operators with constant coefficients, Funktsional. Anal. i Prilozhen. 3 (1969), no. 3, 1-16; English transl., Funct. Anal. Appl. 3 (1969), no. 3, 167-180. MR.0253083 (40:6298)

[32] I. Ts. Gohberg and M. G. Krein, Introduction to the theory of linear non-selfadjoint operators in Hilbert space, "Nauka", Moscow, 1965; English transl., Amer. Math. Soc., Providence, RI, 1969. MR0220070 (36:3137)

[33] J. Weidmann, Spectral theory of ordinary differential operators, Lecture Notes in Math., vol. 1258, Springer-Verlag, Berlin, 1987. MF 0923320 (89b:47070)

[34] V. Vogelsang, Selfadjoint extensions of Dirac operators for nonspherically symmetric potentials in Coulomb scattering, Integral Equations Operator Theory 10 (1987), 841-858. MR0911995 (89b:35133)

[35] G. Nenciu, Distinguished self-adjoint extension for Dirac operator with potential dominated by multicenter Coulomb potentials, Helv. Phys. Acta 50 (1977), 1-3. MR0462346 (57:2320)

[36] M. Klaus, Dirac operators with several Coulomb singularities, Helv. Phys. Acta 53 (1980), 463-482 (1981). MR0611770 (83e:81023)

[37] V. Ivrii, Microlocal analysis and precise spectral asymptotics, Springer-Verlag, Berlin, 1998. MR.1631419 (99e:58193)

[38] I. M. Gel'fand, R. A. Minlos, and Z. Ya. Shapiro, Representations of the rotation group and of the Lorentz group, and their applications, Fizmatgiz, Moscow, 1958. (Russian) MR0114876 (22:5694)

[39] V. Ya. Ivrii, Exact spectral asymptotics for elliptic operators acting in vector bundles, Funktsional. Anal. i Prilozhen. 16 (1982), no. 2, 30-38; English transl., Funct. Anal. Appl. 16 (1982), no. 2, 101-108. MR0659163 (84a:58087)

Moscow Institute of Electronics and Mathematics, Moscow 109028, Russia

E-mail address: magran@orc.ru

Department of Mathematics, Chalmers University of Technology, Göteborg 41296, SweDEN

E-mail address: grigori@math.chalmers.se

Received 20/SEP/2003

Translated by V. I. VASYUNIN 